\title{
Base Flow Investigation of the Apollo Command Module in the Frame of AVT-136
}

\author{
Louis. M.G. Walpot ${ }^{1}$ \\ AOES Netherlands BV, The Netherlands \\ Peter Noeding ${ }^{2}$ \\ EADS Astrium Bremen, Germany \\ Ferry Schrijer ${ }^{3}$ \\ TU Delft, The Netherlands \\ and \\ Michael J. Wright ${ }^{4}$ \\ NASA Ames Research Center, Moffett Field, CA 94035, USA
}

\begin{abstract}
The current uncertainty levels for afterbody (base) aeroheating are very large for capsule like (re)entry vehicles. The design margin on missions have traditionally ranged up to $200 \%$ of CFD predictions. These uncertainties can be attributed to a lack of validation of the CFD tools with suitable flight data and a general difficulty in simulating afterbody flow fields in ground facilities in which base flows are perturbed by sting effects. In addition to basic smooth wall afterbody properties, local disturbances, e.g. connectors, windows and RCS thruster/flowfield interactions have to be taken into account for design. Without an established confidence in the ability of CFD to predict the base flowfield, any attempt to predict the latter effects would be futile. Within RTO AVT-136 / RTG-043 a task group has been created to investigate base flows of the AS-202. The AS-202 was one the four full-scale Apollo flight tests in 1966 at super-orbital entry speeds (in excess of $9 \mathrm{~km} / \mathrm{s}$ ), prior to manned missions. This paper deals with the CFD analysis of the hot hypersonic AS-202 flight and supersonic tests of a scaled AS-202 model in the TU Delft supersonic wind tunnel. CFD results of European and US structured and unstructured codes will be analyzed and compared with the AS-202 flight data and the available wind tunnel data of the TU Delft.
\end{abstract}

\section{Introduction}

$\mathrm{I}^{\mathrm{N}}$ $\mathrm{N}$ recent years, both Europe and the US are developing hypersonic research and operational vehicles. These include (re)entry capsules (both ballistic and lifting) and lifting bodies such as ExoMars, EXPERT, ARV, CEV and IXV. The research programs are meant to enable technology and engineering capabilities to support during the next decade the development of affordable (possibly reusable) space transportation systems as well as hypersonic weapons systems for time critical targets. These programs have a broad range of goals, ranging from the qualification of thermal protection systems, the assessment of RCS performances, the development of GNC algorithms, to the full demonstration of the performance and operability of the integrated vehicles. Since the aerothermodynamic characteristics influence nearly all elements of the vehicle design, the accurate prediction of the aerothermal environment is a pre-requisite for the design of efficient hypersonic systems. Significant uncertainties in the prediction of the hypersonic aerodynamic and the aerothermal loads can lead to conservative margins in the design of the vehicle including its Outer Mould Line (OML), thermal protection system, structure, and required

\footnotetext{
${ }^{1}$ Senior aerothermodynamicist, Aerospace Department, Huygensstraat 34, Noordwijk, AIAA Member .

${ }_{2}^{2}$ Aerospace Engineer, Fluidics and Aerothermodynamics, Airbus-Allee 1, D-28199 Bremen, AIAA Member.

${ }^{3}$ Assistant Professor, High Speed aerodynamics, Kluyverweg 1, Delft.

${ }^{4}$ Senior Research Scientist, Reacting Flow Environments Branch, MS 230-2, Senior Member.
} 
control system robustness. The current level of aerothermal prediction uncertainties results therefore in reduced vehicle performances (e.g. suboptimal payload to mass ratio, increased operational constraints).

On the other hand, present computational capabilities enable the simulation of three dimensional flow fields with complex thermo-chemical models over complete trajectories and ease the validation of these tools by e.g. reconstruction of detailed wind tunnel tests performed under identified and controlled conditions (flow properties and vehicle attitude in particular). These controlled conditions are typically difficult to achieve when performing in flight measurements which in turn results in large associated measurement uncertainties. Similar problems arise when attempting to rebuild measurements performed in "hot" ground facilities, where the difficulty level is increased by the addition of the free-flow characterization itself. The implementation of ever more sophisticated thermochemical models is no obvious cure to the aforementioned problems since their effect is often overwhelmed by the large measurement uncertainties incurred in both flight and ground high enthalpy facilities.

Concurrent to the previous considerations, a major contributor to the overall vehicle mass of re-entry vehicles is the afterbody thermal protection system. This is due to the large acreage (equal or bigger than that of the forebody) to be protected. The present predictive capabilities for base flows are comparatively lower than those for windward flowfields and offer therefore a substantial potential for improving the design of future re-entry vehicles. To that end, it is essential to address the accuracy of high fidelity CFD tools exercised in the US and EU, which motivates a thorough investigation of the present status of hypersonic flight afterbody heating.

This paper addresses the predictive capabilities of after body flow fields of re-entry vehicles investigated in the frame of the NATO/RTO - RTG-043 task group and is structured as follows:

First, the verification of base flow topologies on the basis of available wind-tunnel results performed under controlled supersonic conditions (i.e. cold flows devoid of reactive effects) is performed. Such tests address the detailed characterization of the base flow with particular emphasis on separation/reattachment and their relation to Mach number effects. The tests have been performed on an Apollo-like re-entry capsule configuration.

Second, the tools validated in the frame of the previous effort are exercised and appraised against flight-test data collected during the Apollo AS-202 re-entry.

\section{Experimental Data}

\section{A. Experimental supersonic data}

Ground tests were performed to obtain flow field data of the AS-202 configuration under supersonic test conditions. Shadowgraph measurements provided insight into shock and shear layer patterns found under different angles of attack at Mach numbers between 2 and 4. The facility used in the ground experiments is the TST27 transonic/supersonic blow-down wind tunnel located at TU Delft in The Netherlands, (see Figure 1). This tunnel features a $27 \times 28 \mathrm{~cm} 2$ test section and sports two flexible nozzle walls that allow to continuously vary the Mach number between 0.5 and 4.2. The total pressure in the settling chamber can be varied from 2 bar at Mach 0.5 to 20 bar at Mach 4.2, which results in a unit Reynolds number range from $25 \times 10^{6}$ to $150 \times 10^{6}$. The maximum run-time of the facility is $300 \mathrm{~s}$. Two $30 \mathrm{~cm}$ diameter schlieren windows in the side of the test section are available for optical access. In the current experiments, the wind tunnel was operated in the Mach 2 to 4 range with a total pressure ranging from $2.7-12$ bar and a total temperature of $288 \mathrm{~K}$. The capsule geometry used for the definition of the wind tunnel model is a scaled version of the AS-202 outer mold line as defined in Figure 1. The model has a diameter of $50 \mathrm{~mm}$ and is fabricated out of stainless steel, it is side-mounted on a stainless steel blade sting. Two models are used with mounting at respectively 0 and 25 degrees, (see Figure 1). Angles of attack other than 0 or 25 degrees were reached by deflecting the mounting sting according to the sought flow condition. 

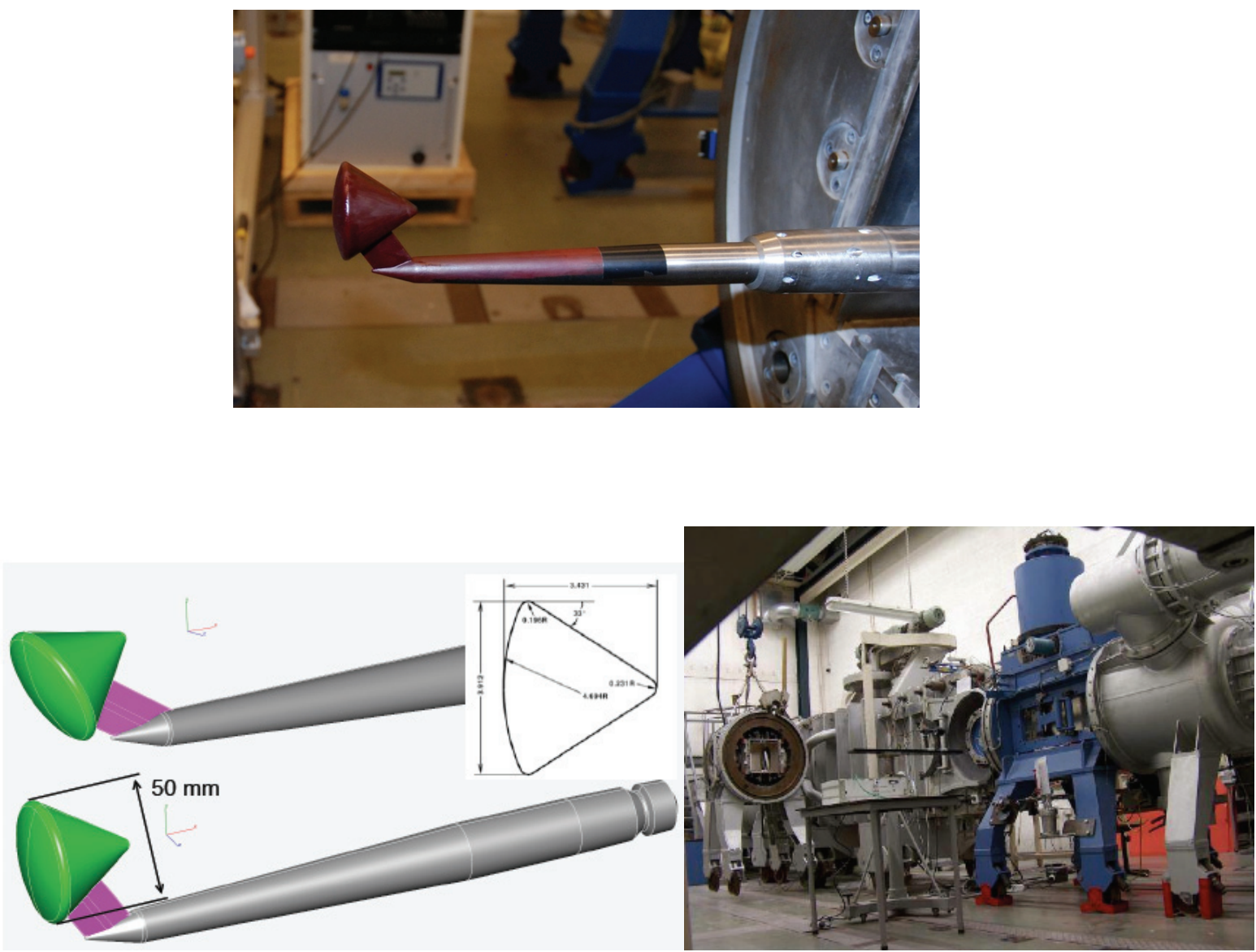

Figure 1 The $5 \mathrm{~cm}$ diameter AS202 for 0 and $25 \mathrm{deg}$ in the TU Delft TST27 wind tunnel.

Shadowgraphs were obtained during the tests using a 3872 × 2592 pixel Nikon D80 camera. The illumination was provided by a Xenon spark light with an emission time of $20 \mathrm{~ns}$, effectively freezing the flow in a single snapshot. In addition to the shadowgraph images.

\section{B. AS-202 flight test}

The flight data used for assessment/comparison of heat flux data on the capsule were taken from the AS-202 flight test $^{123}$, which was performed as part of the Apollo program. Once the Apollo entry vehicle design was determined, two flight tests of the actual Command Module (AS-201 \& AS-202) were conducted at super-orbital entry velocities resulting from suborbital boosted trajectories with an intentional skip manoeuvre. Although AS-201 did not carry an onboard inertial measurement unit (IMU), one was carried during the AS-202 flight, which enabled a reconstruction of the flight trajectory and vehicle orientation as a function of time.

In this paper the afterbody heating environment for the Apollo Command Module shape as measured on the AS-202 mission is used as basis for comparison between CFD results and flight data.

Figure 2 shows the outer mould line of the AS-202 as modelled for the CFD analyses. The re-entry trajectory of AS202 in terms of velocity and altitude vs. time is shown in Figure 3 . The points in time and the related free-stream conditions used for comparison to flight data are tabulated in

Table 1. The small side slip angle has been neglected in the current simulations. The locations of calorimeters used to determine the heat fluxes on the AS-202 conical afterbody are depicted in Figure 4. Table 2 contains the exact coordinates of each calorimeter position. Figure 2, 3, 4 and Table 1 and 2 are taken from Ref Wright et al ${ }^{4}$. 


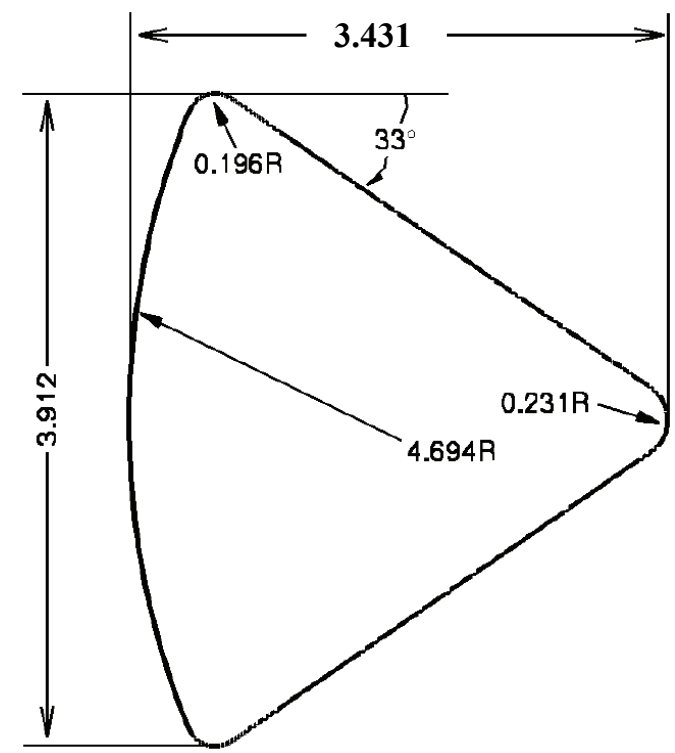

Figure 2 Schematic drawing of the outer mold line of AS-202 capsule as modeled in this work.

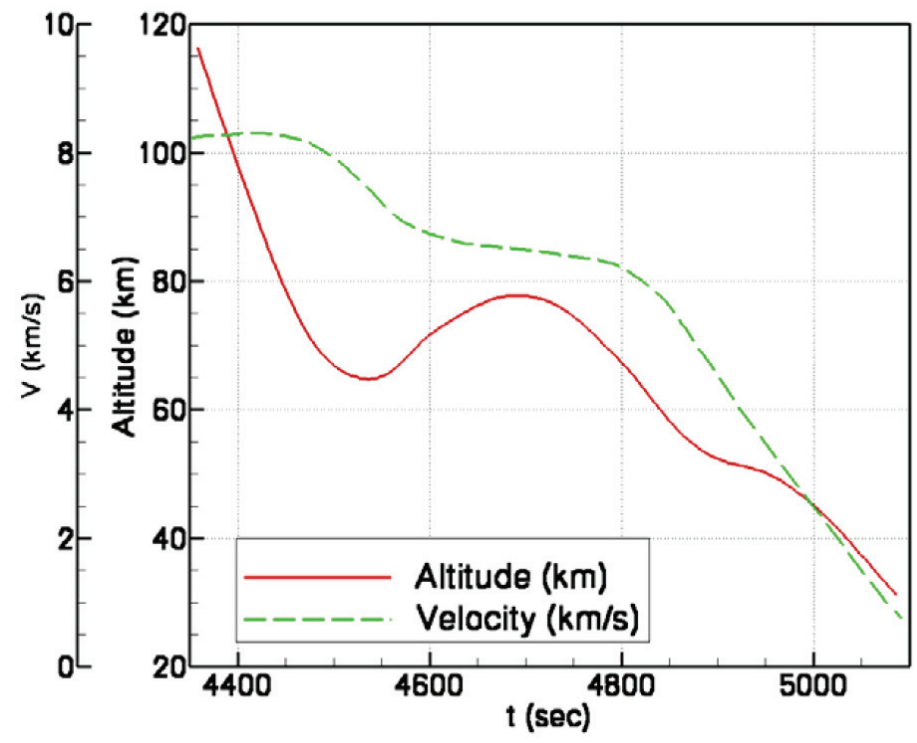

Figure 3. Altitude and velocity as a function of time from launch for AS-202

Table 1: AS-202 Trajectory points and freestream conditions.

\begin{tabular}{ccccccccc}
\hline \hline $\begin{array}{c}\text { Time }^{\mathbf{a}} \\
(\mathbf{s})\end{array}$ & $\begin{array}{c}\text { Alt. } \\
(\mathbf{k m})\end{array}$ & $\mathbf{R e}_{\mathbf{D}}{ }^{\mathbf{b}}$ & $\begin{array}{c}\mathbf{V} \\
(\mathbf{k m} / \mathbf{s})\end{array}$ & $\mathbf{M}$ & $\begin{array}{c}\boldsymbol{\rho}_{\infty} \\
\left(\mathbf{k g} / \mathbf{m}^{3}\right)\end{array}$ & $\begin{array}{c}\boldsymbol{T}_{\infty} \\
(\mathbf{K})\end{array}$ & $\begin{array}{c}\boldsymbol{\alpha} \\
(\mathbf{d e g})\end{array}$ & $\begin{array}{c}\boldsymbol{\beta} \\
(\mathbf{d e g})\end{array}$ \\
\hline & & & & & & & & \\
4455 & 76.8 & $7.5 \times 10^{4}$ & 8.24 & 28.6 & $3.38 \mathrm{e}-5$ & 205 & 18.2 & 2.0 \\
4475 & 71.3 & $1.8 \times 10^{5}$ & 8.15 & 27.6 & $8.76 \mathrm{e}-5$ & 217 & 17.9 & 2.5 \\
4500 & 70.0 & $3.0 \times 10^{5}$ & 7.92 & 26.2 & $1.52 \mathrm{e}-4$ & 227 & 17.8 & 2.5 \\
4510 & 66.0 & $3.2 \times 10^{5}$ & 7.80 & 25.6 & $1.69 \mathrm{e}-4$ & 230 & 17.8 & 2.5 \\
4530 & 64.9 & $3.4 \times 10^{5}$ & 7.53 & 24.5 & $1.84 \mathrm{e}-4$ & 234 & 17.9 & 2.5 \\
4560 & 66.0 & $2.7 \times 10^{5}$ & 7.07 & 23.2 & $1.53 \mathrm{e}-4$ & 231 & 18.1 & 2.5 \\
4600 & 71.6 & $1.3 \times 10^{5}$ & 6.74 & 22.9 & $7.19 \mathrm{e}-5$ & 215 & 18.3 & 2.5 \\
4650 & 76.2 & $5.7 \times 10^{4}$ & 6.56 & 22.8 & $3.24 \mathrm{e}-5$ & 206 & 18.5 & 2.0 \\
4700 & 77.2 & $4.3 \times 10^{4}$ & 6.49 & 22.7 & $2.45 \mathrm{e}-5$ & 203 & 18.5 & 2.0 \\
4750 & 74.5 & $7.6 \times 10^{4}$ & 6.39 & 22.0 & $4.50 \mathrm{e}-5$ & 210 & 18.4 & 2.0 \\
4800 & 67.3 & $2.1 \times 10^{5}$ & 6.21 & 20.5 & $1.37 \mathrm{e}-4$ & 210 & 18.4 & 2.0 \\
4825 & 62.9 & $3.5 \times 10^{5}$ & 5.97 & 19.2 & $2.81 \mathrm{e}-4$ & 239 & 18.3 & 2.0 \\
4850 & 58.2 & $5.3 \times 10^{5}$ & 5.62 & 17.6 & $4.14 \mathrm{e}-4$ & 252 & 18.3 & 2.5 \\
4875 & 54.6 & $6.9 \times 10^{5}$ & 5.07 & 15.6 & $6.16 \mathrm{e}-4$ & 262 & 18.4 & 2.5 \\
4900 & 52.4 & $7.6 \times 10^{5}$ & 4.53 & 13.2 & $8.00 \mathrm{e}-4$ & 268 & 18.6 & 2.5 \\
\hline \hline
\end{tabular}

${ }^{\mathrm{a}}$ Seconds after launch. ${ }^{\mathrm{b}}$ Freestream Reynolds number based on body diameter. 


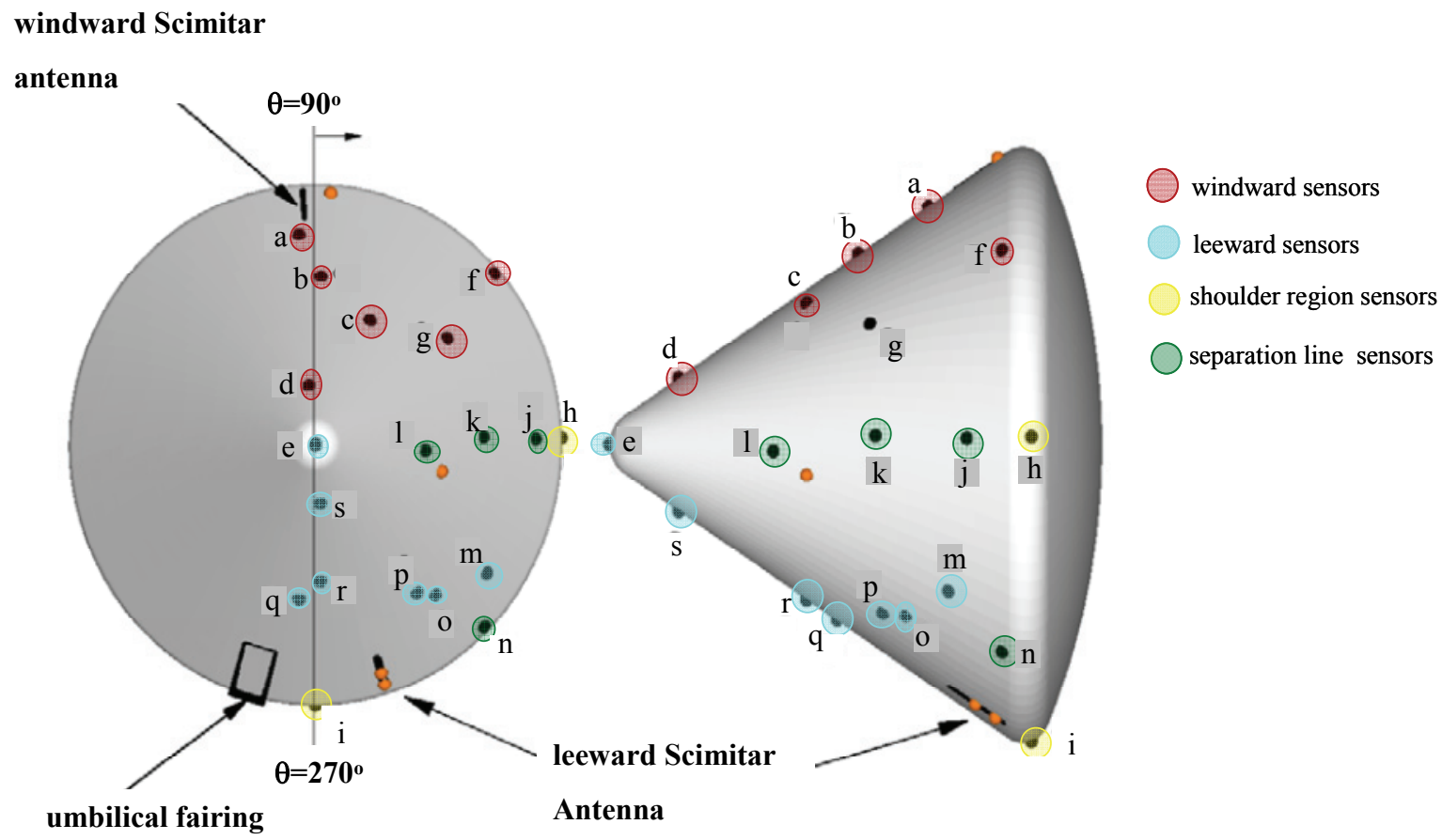

Figure 4: Locations of calorimeters on AS-202 conical afterbody. Orange symbols indicate inoperative instruments. Letters correspond to the ID

Table 2 Afterbody calorimeter locations for AS-202.

\begin{tabular}{cccccccc}
\hline \hline $\mathbf{I D}^{\mathbf{a}}$ & $\begin{array}{c}\mathbf{X}^{\mathbf{b}} \\
(\mathbf{c m})\end{array}$ & $\begin{array}{c}\boldsymbol{\theta}^{b} \\
(\mathbf{d e g})\end{array}$ & $\begin{array}{c}\text { Range } \\
\left(\mathbf{W} / \mathbf{c m}^{2}\right)\end{array}$ & $\mathbf{I D}^{\mathbf{a}}$ & $\begin{array}{c}\mathbf{X}^{\mathbf{b}} \\
(\mathbf{c m})\end{array}$ & $\begin{array}{c}\boldsymbol{\theta}^{\boldsymbol{b}} \\
(\mathbf{d e g})\end{array}$ & $\begin{array}{c}\text { Range } \\
\left(\mathbf{W} / \mathbf{c m}^{2}\right)\end{array}$ \\
\hline & & & & & & & \\
- & 72.6 & 93.7 & $0-114$ & 1 & 228.8 & 182.9 & $0-28$ \\
$\mathrm{a}$ & 120.8 & 85.3 & $0-57$ & $\mathrm{~m}$ & 106.8 & 215.3 & $0-11.4$ \\
$\mathrm{~b}$ & 169.8 & 92.0 & $0-57$ & $\mathrm{n}$ & 69.5 & 225.5 & $0-11.4$ \\
$\mathrm{c}$ & 205.6 & 115.0 & $0-57$ & - & 205.6 & 191.3 & $0-28$ \\
$\mathrm{~d}$ & 294.8 & 83.4 & $0-57$ & $\mathrm{o}$ & 136.6 & 229.8 & $0-11.4$ \\
$\mathrm{e}$ & 343.1 & $\mathrm{Apex}$ & $0-28$ & $\mathrm{p}$ & 152.6 & 234.0 & $0-28$ \\
$\mathrm{f}$ & 69.5 & 138.0 & $0-57$ & $\mathrm{q}$ & 184.3 & 276.4 & $0-28$ \\
$\mathrm{~g}$ & 161.5 & 142.8 & $0-28$ & $\mathrm{r}$ & 205.6 & 267.8 & $0-28$ \\
$\mathrm{~h}$ & 54.5 & 178.5 & $0-28$ & $\mathrm{~s}$ & 294.8 & 265.0 & $0-28$ \\
$\mathrm{i}$ & 54.5 & 270.0 & $0-11.4$ & - & 74.1 & 253.0 & $0-28$ \\
$\mathrm{j}$ & 94.1 & 178.6 & $0-28$ & - & 88.0 & 253.0 & $0-57$ \\
$\mathrm{k}$ & 157.6 & 177.5 & $0-28$ & & & & \\
\hline \hline
\end{tabular}

${ }^{\mathrm{a}}$ Corresponds to Fig. 17 in Ref. 1. Those without letters were non-functional during AS-202.

${ }^{\mathrm{b}}$ Refer to Figure 4 for coordinate system definition. 


\section{Computational Modeling}

AOES used the LORE ${ }^{5}$ CFD code for the Navier-Stokes calculations presented in this paper. For a number of computational conditions, including wind tunnel and AS-202 flight test data, Navier-Stokes calculations have also been performed by ASTRIUM Bremen using the TAU code ${ }^{6}$. These results are hereafter compared to the test data and the LORE results. Finally, the existing CFD Results (DPLR CFD-Code) published by Wright et al. ${ }^{4}$ for the AS202 flight test data are also reported here for comparison.

LORE is a multi-block finite-volume Navier-Stokes solver augmented with finite rate chemistry and thermal nonequilibrium effects. For the present effort, turbulence effects have been modeled with Menter's SST (Shear Stress Transport) and Baldwin-Lomax models. LORE has been extensively validated against X-38 wind tunnel test data (obtained at ONERA S2Ma, Langley Research Center (LaRC) $16 \mathrm{ft}$ and FFA's T1500) ${ }^{5}$ and CARV ${ }^{7}$.

For the LORE calculations presented here super-catalytic wall boundary conditions have been used, which represents a conservative approach often used for design. In this assumption the wall composition is forced to be equal to the freestream. This boundary condition is conservative in that the maximum chemical enthalpy is recovered at the wall, but it does not account for potential rate-limiting processes in the underlying surface chemistry (surface reaction rates are taken to be infinite). In the current flight test cases ranging from Mach 28 to Mach 4, it has been observed that there is no appreciable difference in base flow heating between supercatalytic and diffusion limited with LORE.

The DLR Navier-Stokes- and Euler-Solver TAU-Code ${ }^{6}$ has been used at EADS Astrium Bremen. The code has been extensively validated against test results and other CFD Codes in the past, see e.g. ${ }^{7}$. The three-dimensional CFD program was developed by the German Aerospace Center DLR for unstructured and structured grids (under participation of several branches of EADS Germany). The TAU flow solver represents a three-dimensional parallel hybrid multi-grid code employing a finite volume scheme for solving the Reynolds-averaged Navier-Stokes equations. The inviscid fluxes are calculated using an AUSM or a Roe type 2nd-order upwind scheme. The gradients of the flow variables are determined by employing a Green-Gauss formula. Central differences are used to discretize the viscous fluxes. Treatment of viscous walls within the TAU-Code allows for adiabatic, constant wall temperature or radiation equilibrium conditions.

Turbulence modeling: The TAU Code offers a choice of different one- and two-equation turbulence models (Spalart-Allmaras-model, various versions of the k- $\omega$-model). For AS-202, the Spalart-Allmaras model was used to cover turbulent flow situations.

Air Chemistry: Regarding the available thermo-chemical models for hypersonic flows, the following options are incorporated into the TAU-Code:

a) Equilibrium chemistry: Air is considered as a five species ideal gas mixture. The temperature and pressure dependent equilibrium gas properties are modeled via appropriate fit functions. A temperature range between $50 \mathrm{~K}$ and $20000 \mathrm{~K}$ and a density range between $10-12 \mathrm{~kg} / \mathrm{m}^{3}$ and $10 \mathrm{~kg} / \mathrm{m}^{3}$ is covered by the fits currently in use.

b) Chemical nonequilibrium: The nonequilibrium model currently implemented in the TAU-Code consists of a five species and seventeen reactions air model employing the finite reaction rates according to Gupta et al. This can be easily replaced by more detailed models ${ }^{8}$. The diffusion is modeled according to Fick's law by a single diffusion coefficient for all species. The diffusion coefficient is connected to the local viscosity via a user-specified constant Schmidt number. Within AS-202 flow simulations chemical nonequilibrium in conjunction with a fully catalytic wall boundary condition was assumed (as opposed to the supercatalytic wall conditions in the LORE calculations. However, for the conditions considered here the dependency of the the wall heat fluxes on either wall condition is considered small).

The CFD modeling published earlier, using the DPLR code for comparison to the AS-202 flight data is described in detail in ${ }^{4}$. DPLR is a parallel multi-block finite volume code that solves the reacting Navier-Stokes equations including finite-rate chemistry and the effects of thermal nonequilibrium. The Euler fluxes are computed using a modified (low-dissipation) form of Steger-Warming flux vector splitting, with third-order spatial accuracy obtained via MUSCL extrapolation. Viscous fluxes are computed to second-order accuracy using a central difference approach. 


\section{CFD Grids:}

Some TAU Code Calculations employed the same structured grids as used by LORE, so as to facilitate direct code comparison. Other TAU CFD meshes for the AS-202 analyses employed a hybrid grid approach consisting of structured prismatic grid layers in the wall regions to resolve the boundary layers as well as tetrahedral cells covering the rest of the computational domain. For the hybrid grids, in order to limit the impact of the grid density on the computed flow-field, the solution-dependent grid adaptation features of the TAU Code were used. Typically, a total of 2 to 3 grid adaptation cycles were performed to improve the solutions. Figure 5 shows the hybrid TAU grid after two adaptation cycles. Shock and shear layer structures are clearly reflected by the locally adapted/refined regions of the mesh. The lower left insert of Figure 5 shows the complete computational domain. The upper left insert shows the "structured" boundary layer mesh part in the shoulder region.
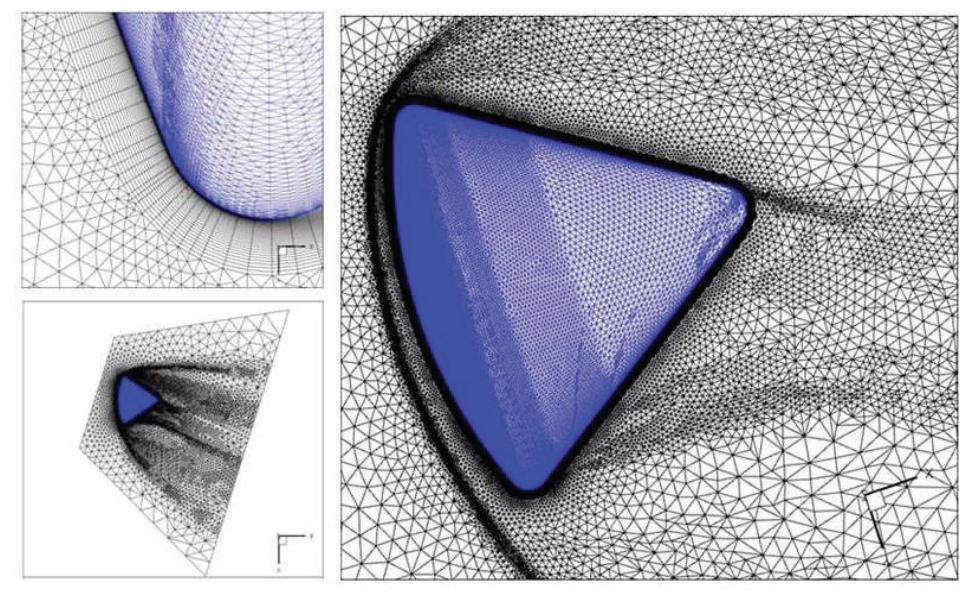

Figure 5 : Hybrid TAU-Code CFD grid for the AS 202 flight test geometry

The structured grids have been generated with GridPro. The meshes are featured with smoothness and are each shock adapted. The meshes for flight and wind tunnel w/o sting comprise 76 blocks and roughly 8 million cells. The meshes with sting have 151 blocks with 24 million cells, resp. 3 million cells for the undoubled mesh.. In Figure 6 each other point has been plotted. The smallest distance at the wall is $1 . e-6 \mathrm{~m}$. Grid convergence is checked by comparing the solution in terms of separation /flow topologies for the WT mode and heat fluxes in front and base for the flight cases. It can bee seen in Figure 7, where the surface meshes are plotted for the fine and undoubled mesh, that the pressure contour lines on the front shield are independent of the mesh. Table 3 summarizes main differences in the modeling approaches for the AS-202 calculations between the LORE, TAU Code and DPLR analyses.
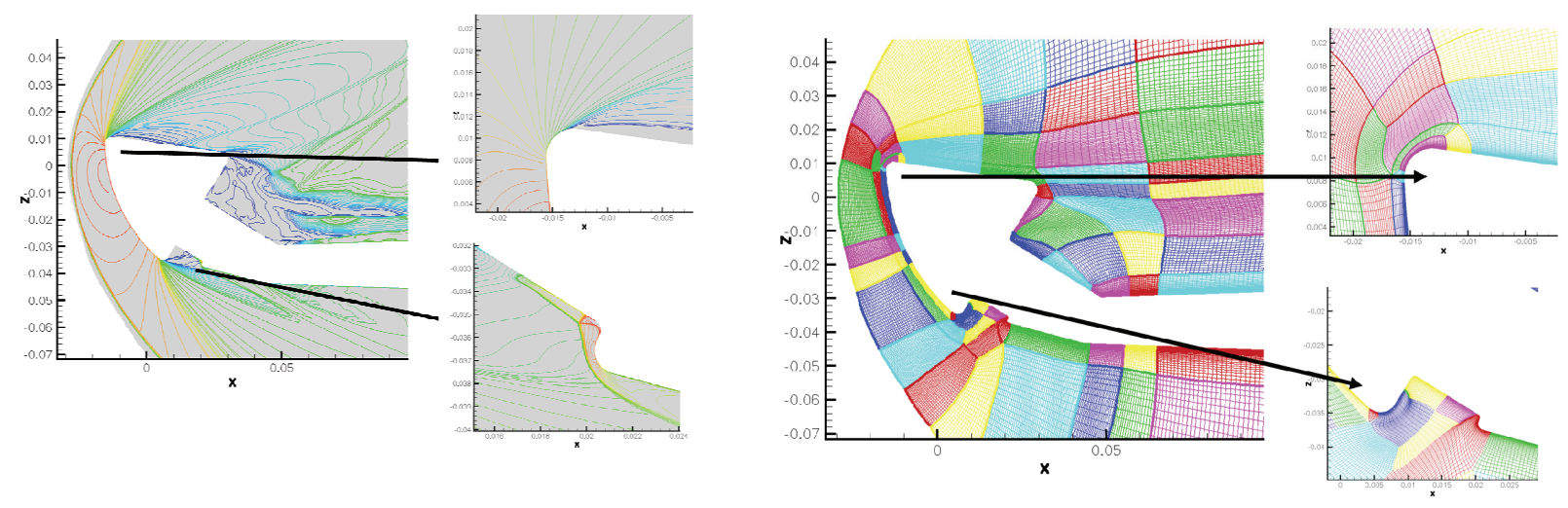

Figure 6: Structured grid used both by LORE and TAU for the TST27 supersonic tests 

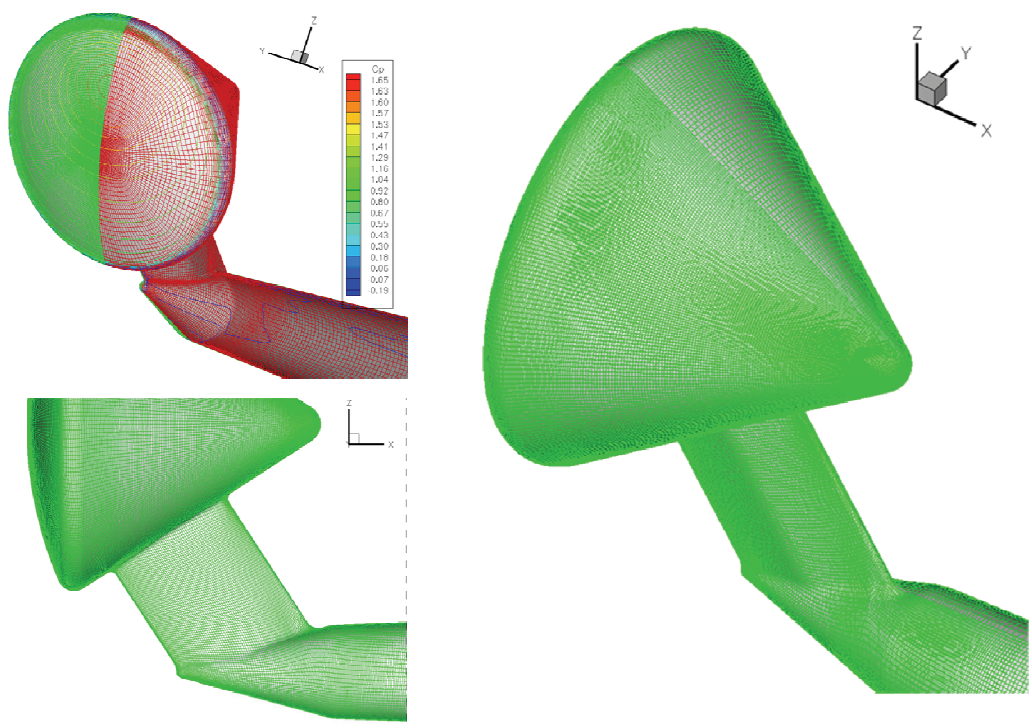

Figure 7. : Structured grid used both by LORE and TAU for the TST27 supersonic tests

Table 3. : Differences in modeling of the AS202 Flight Case: LORE, TAU Code, DPLR

\begin{tabular}{|c|c|c|c|c|c|}
\hline & CFD Meshes & $\begin{array}{l}\text { thermochemical } \\
\text { Model }\end{array}$ & $\begin{array}{l}\text { Transport } \\
\text { Coefficients }\end{array}$ & Wall Catalycity & $\begin{array}{l}\text { Turbulence } \\
\text { Model }\end{array}$ \\
\hline LORE & $\begin{array}{l}\text { block structured } \\
\text { Level 1: } 1 \text { Mio. } \\
\text { Cells } \\
\text { Level 2: } 8 \text { Mio. } \\
\text { Cells }\end{array}$ & $\begin{array}{l}\text { thermochemical } \\
\text { nonequilibrium } \\
5 \text { Species Finite } \\
\text { Rate(Park 1993) }\end{array}$ & $\begin{array}{l}\text { Constant Lewis } \\
\text { number Le }=1.2 \\
\text { SCEBD }\end{array}$ & Super catalytic & $\begin{array}{l}\text { Baldwin- } \\
\text { Lomax }\end{array}$ \\
\hline TAU-Code & $\begin{array}{l}\text { Structured } \\
1 \text { Mio. Cells(as } \\
\text { Level 1/LORE), or } \\
\text { Solution adaptive, } \\
\text { Hybrid/Unstructured } \\
4 \text { Mio. Cells }\end{array}$ & $\begin{array}{l}\text { Chemical } \\
\text { Nonequilibrium } \\
5 \text { Species Finite } \\
\text { Rate(Gupta) }\end{array}$ & $\begin{array}{l}\text { Constant } \\
\text { Schmidt Number } \\
\text { for all species } \\
\mathrm{Sc}=0.7\end{array}$ & Fully Catalytic & $\begin{array}{l}\text { Spalart- } \\
\text { Allmaras }\end{array}$ \\
\hline DPLR & $\begin{array}{l}\text { block structured } \\
1.5 \text { Mio. Cells }\end{array}$ & $\begin{array}{l}\text { thermochemical } \\
\text { nonequilibrium } \\
5 \text { Species Finite } \\
\text { Rate(Park 1993) }\end{array}$ & $\begin{array}{l}\text { mixing mules } \\
\text { (Gupta) } \\
\text { Diffusion } \\
\text { Coefficients via } \\
\text { SCEBD }\end{array}$ & $\begin{array}{l}\text { diffusion } \\
\text { limited }\end{array}$ & $\begin{array}{l}\text { Baldwin- } \\
\text { Lomax }\end{array}$ \\
\hline
\end{tabular}




\section{Results}

\section{A. Shadowgraphy results from the TST27 Wind Tunnel and related CFD calculations}

General flow pattern and shear layer separation at the capsule shoulder was investigated for various combinations of angle of attack and Mach number. Mach 2 and Mach 4 wind tunnel results and related CFD calculations for varying angles of attack are used to examine the flow topology with respect to:

- $\quad$ Shock patterns: Bow shock, shoulder region, wake areas

- Shock-shock interactions/flow interferences due to the presence of a wind tunnel blade mounting

- Local separation and reattachment of the capsule flow.

- Characteristic flow patterns of the leeside/wake flow.

The quality of the CFD codes with regard to the correct determination of flow separations in the base area is deemed very important in view of the accurate prediction of aerothermal heat fluxes on vehicle leeside during re-entry. The shadowgraphy images from the wind tunnel represent a useful basis for comparison here.

The shadowgraphy images taken for various angle of attack/Mach number combinations are shown in Figure 12 to Figure 16 These figures also contain the corresponding CFD results (calculated density fields), overlaid on the shadowgraphs. As evidenced by Figure 12 to Figure 16, the flow around the capsule is characterized by the bow shock forming in front of the spherical heat shield and by the expansion over the capsule shoulder. The flow overexpands and a lip shock is formed. At smaller angles of attack downstream of the shoulder a completely separated shear layer develops and it does not reattach on the model. The expansion from the low velocity region downstream of the bow shock over the model shoulder is clearly evident. Also the shock coming from the re-compression in the capsule wake is clearly visualized.

With increasing angles of attack it can be observed that the shear layer only partially separates from the upper side of the capsule or stays completely attached at sufficiently large angles of attack. At the model shoulder a small shock wave is present where separation occurs. Further downstream, a stronger shock is formed if and where the shear layer reattaches. Downstream of the capsule a strong shock is present where the wake is re-compressed. The shock emanating from the reattaching shear layer is also evident. The downstream region shows a three dimensional reattachment shock pattern that emanates from the wake behind the capsule.

Oblique shocks are visible at the tip of the lower side of the sting mounting. Shocks are also present at the edge of the mounting, at the location where the cone angle of the sting is reduced. However, no significant interference with the upper side of the capsule model, where the separation behaviour at the cone vs. angle of attack is investigated is apparent from the blade mounting.

The shadowgraphs clearly show whether the flow was attached or separated from the windward side of the capsule. Figure 12 shows a separated and Figure 14 an attached case.

It was found that for increasing Mach numbers, the flow separation occurs at smaller angles of attack. Similar results were obtained by Kruse et al. ${ }^{9}$ In Figure 8, the measurement points are given as a function of angle of attack and Mach number. A blue circle denotes the flow conditions for which the shear layer was found to be attached while the separated cases are represented by a red triangle. In Figure 8 also the curve-fit from Kruse et al. ${ }^{9}$ is shown that forms the border between an attached or a separated shear layer. As can be seen, the separation angle of attack decreases with increasing Mach number. However the angle of attack values found by Kruse et al. ${ }^{9}$ are smaller for Mach 2. This is due to the fact that in those measurements models with sharp-edged shoulders were used, in those cases the separation point is defined and a more or less centered Prandtl-Meyer expansion is formed. In the current experiments, the flow expands more gradually and therefore is likely to separate more easily. 


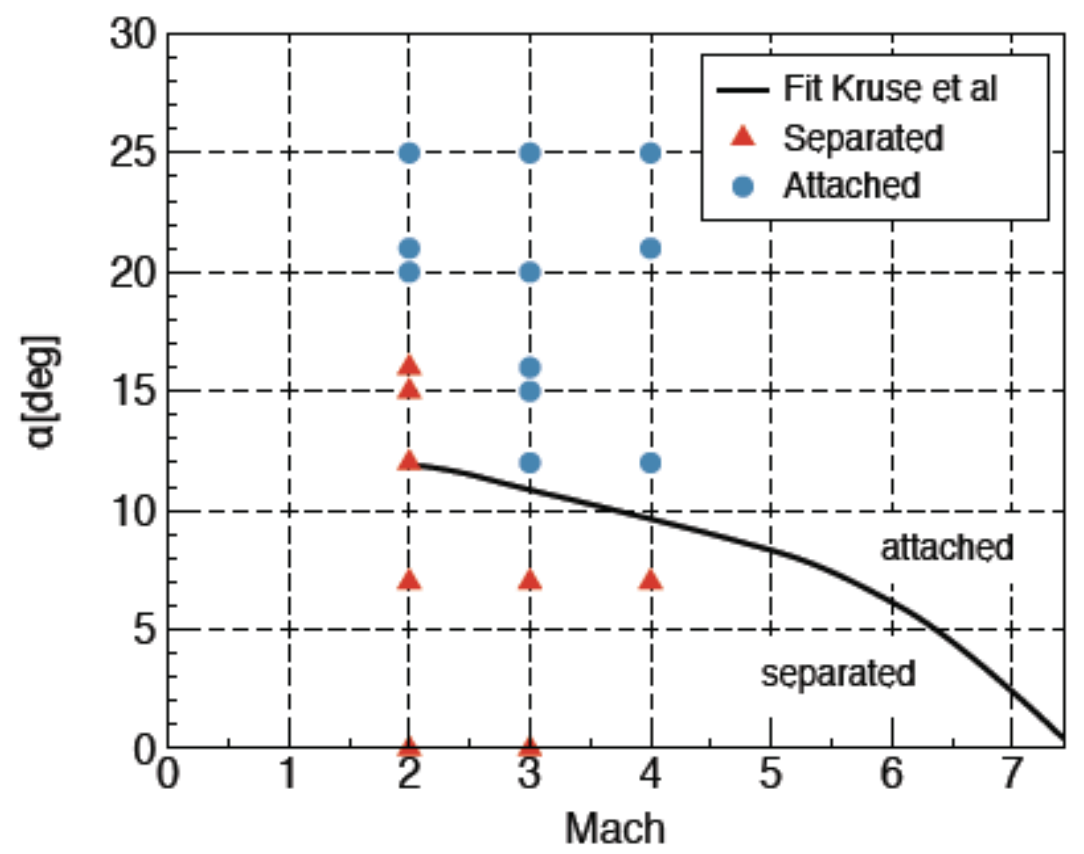

Figure 8.: Boundary layer separation condition as a function of Mach number and angle of attack

Figure 12 to Figure 14 show a series of shadowgraphs for Mach 2 at varying angles of attack between 14 and 22 degrees. At 22 degrees a completely separated shear layer at the upper side of the rear cone of the model can be observed. The separation on the upper side of the cone begins when the angle of attack is reduced to about 19 degrees with a flat separation bubble starting just behind the shoulder with reattachment about halfway along the cone surface. A reattachment shock is visible at the downstream end of the separation bubble. The CFD results show excellent agreement regarding the onset of separation at 19 degree AoA as well as the reattachment position, as evidenced in Figure 13. This is true for the fine ( 8 million cells) as well as for the undoubled (1 million cells) mesh (Figure 9). As confirmed by the related CFD calculations performed without mounting and depicted in

Figure 17 and Figure 18 (where the flow topology is probed downstream of the model by means of planar Mach contour cuts), the presence of the wind tunnel blade/sting does not significantly influence the separation behaviour at the upper side of the cone. This is also evident from the calculated density contours in the symmetry plane, with and without sting/blade, as shown in Figure 11.

The LORE and TAU code calculations properly capture the separation bubble at 19 degrees angle of attack (Figure 10). At $14^{\circ}$ angle of attack, the CFD calculations and related shadowgraphs show a completely separated shear layer on the upper side (Figure 12).

The Mach 4 results at angles of attack $10^{\circ}$ and $14^{\circ}$ (depicted in Figure 15 and Figure 16, respectively) also illustrate excellent agreement between experiment and CFD results. The flow on the upper side is attached at an angle of attack of 14 degrees, whereas it was separated at Mach 2. At an angle of attack of $10^{\circ}$ the flow on the upper side separates at the beginning of the cone without re-attaching.

The wind tunnel tests also indicate a lack of sensitivity of the separation length to the free flow Reynolds numbers within the range reached in the TST27. Therefore it can be reliably assumed that the flow remains laminar for the tested conditions. This is confirmed by the good agreement between the CFD results and the tests, since all calculations considered laminar flow conditions only.

As a conclusion, the CFD methods used have demonstrated their ability to reliably predict the flow pattern including the separation behaviour identified in the shadowgraphy images from the related wind tunnel tests. The qualitative trend found by Kruse ${ }^{4}$ (Figure 8) of decreasing separation tendency with increasing Mach number could be fully confirmed by CFD as well as the wind tunnel tests at TU Delft. 


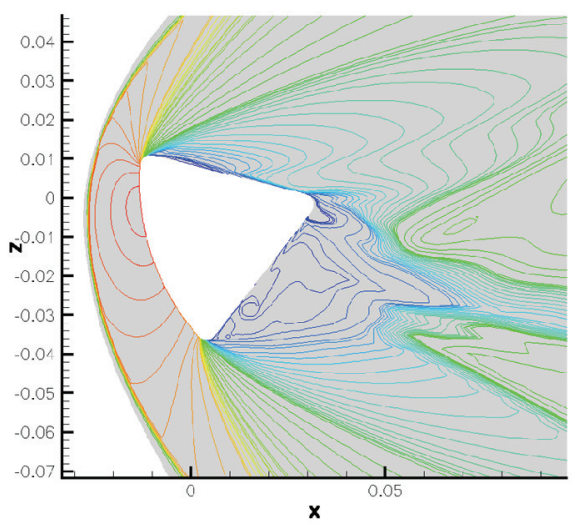

$1 \mathrm{M}$ cells

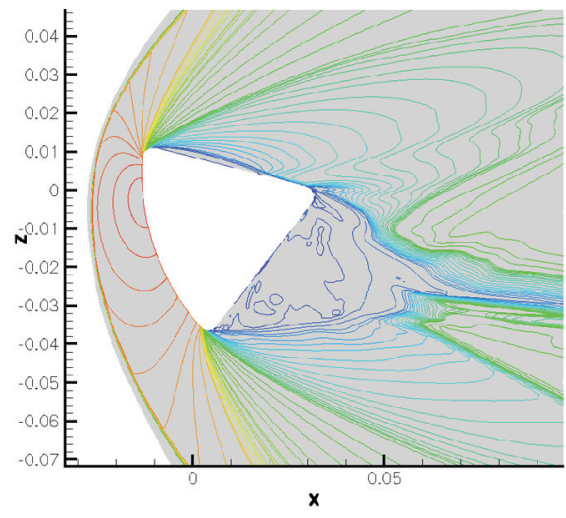

$8 \mathrm{M}$ cells

Figure 9 AS202 in TST27 (without sting) Mach 2 AoA 19 symmetry plane computed with LORE: effect of grid refinement
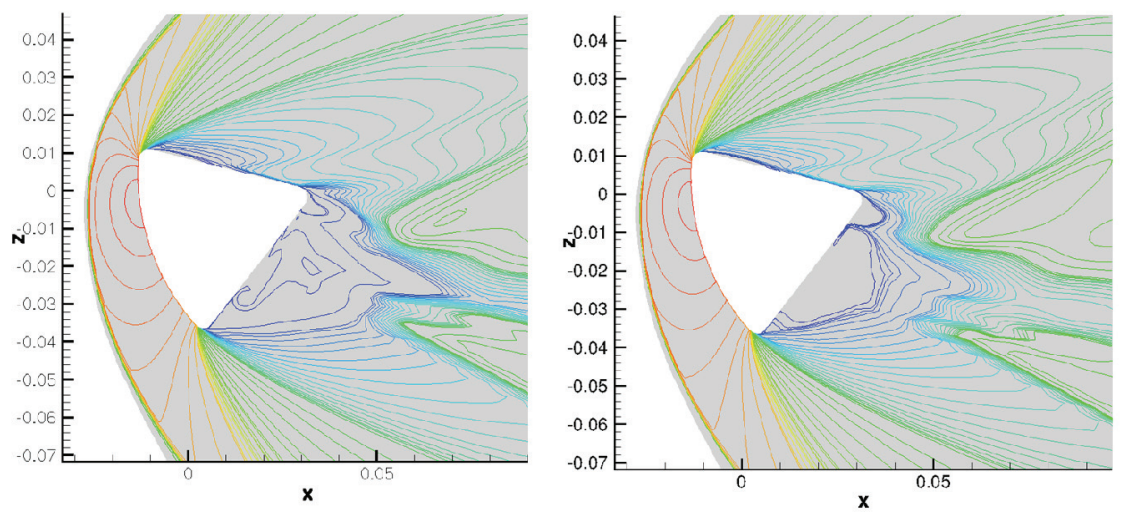

Figure 10 AS202 in TST27 (without sting) Mach 2 AoA 19 symmetry plane computed with LORE and TAU (1M cells)
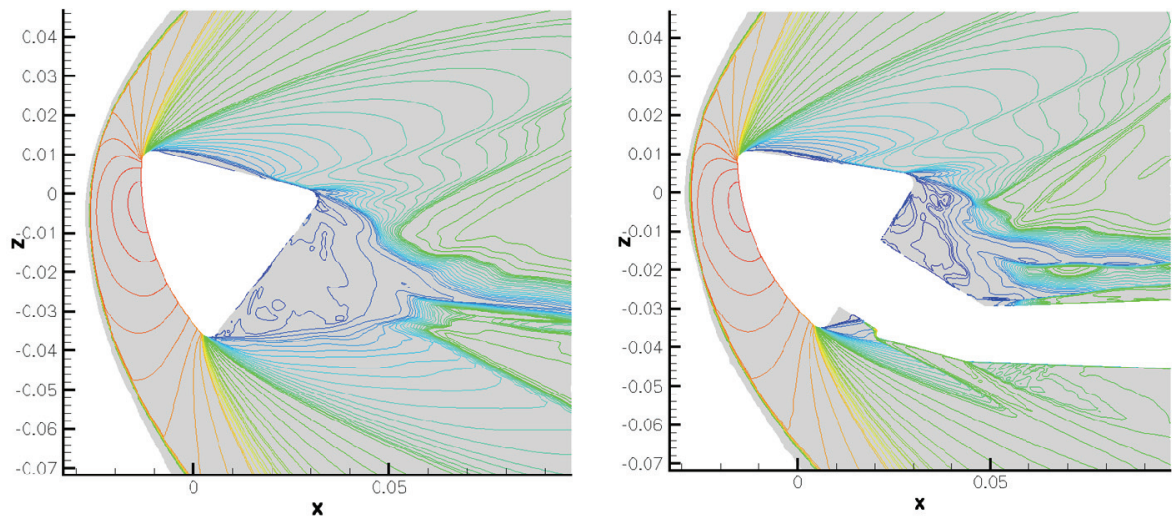

Figure 11 AS202 in TST27 (without sting) Mach 2 AoA 19 symmetry plane computed with LORE: effect of sting /blade vs. no sting/blade on 8Mcells mesh

11

American Institute of Aeronautics and Astronautics 

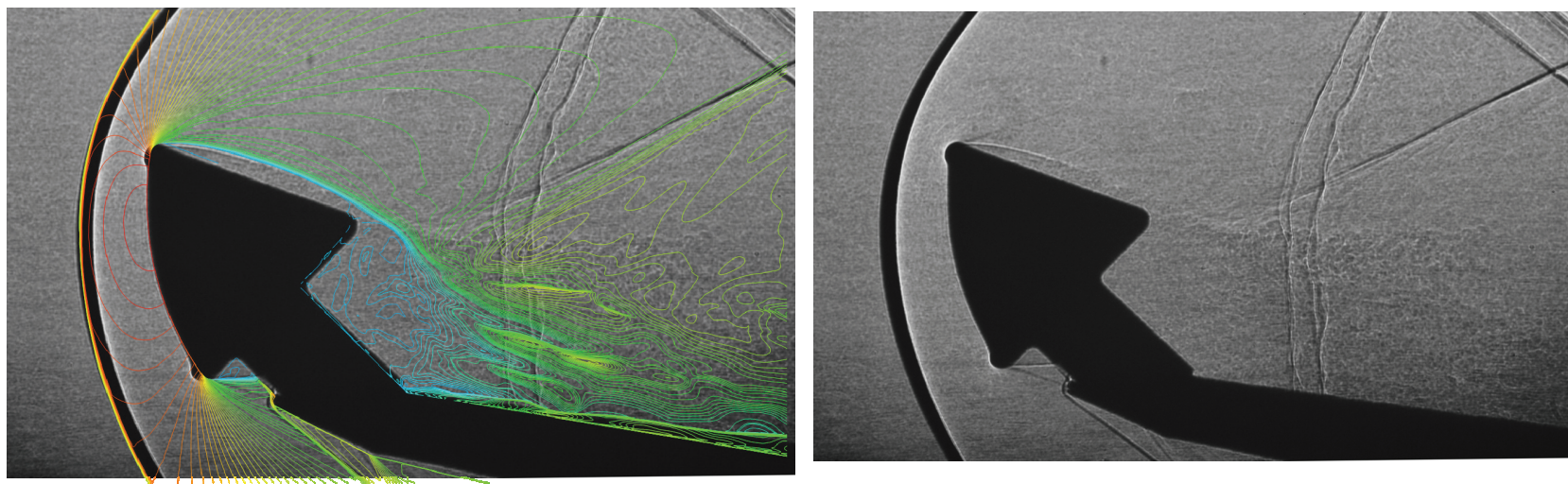

Figure 12: Mach 2 Angle of attack $14 \mathrm{deg}, \mathrm{Pt}=27 \mathrm{Bar}, \mathrm{T} 0=285 \mathrm{~K}$
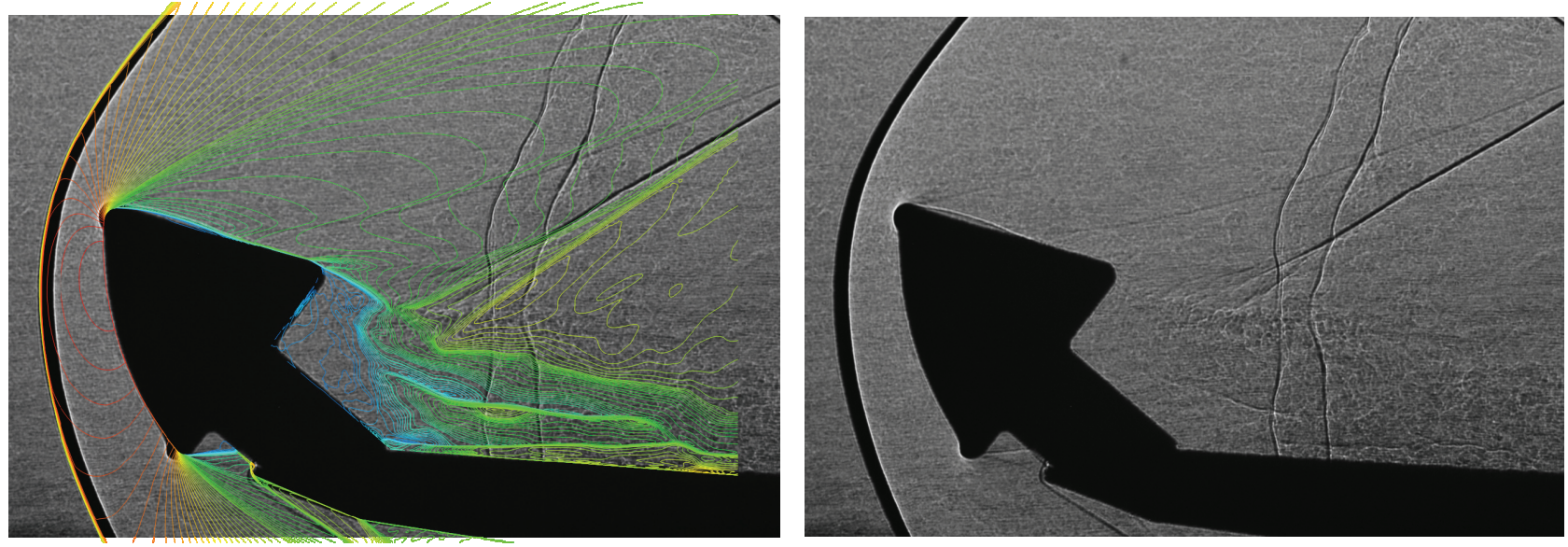

Figure 13: Mach 2: Angle of attack $19 \mathrm{deg}, \mathrm{Pt}=27 \mathrm{Bar}, \mathrm{T0}=\mathbf{2 8 5 K}$
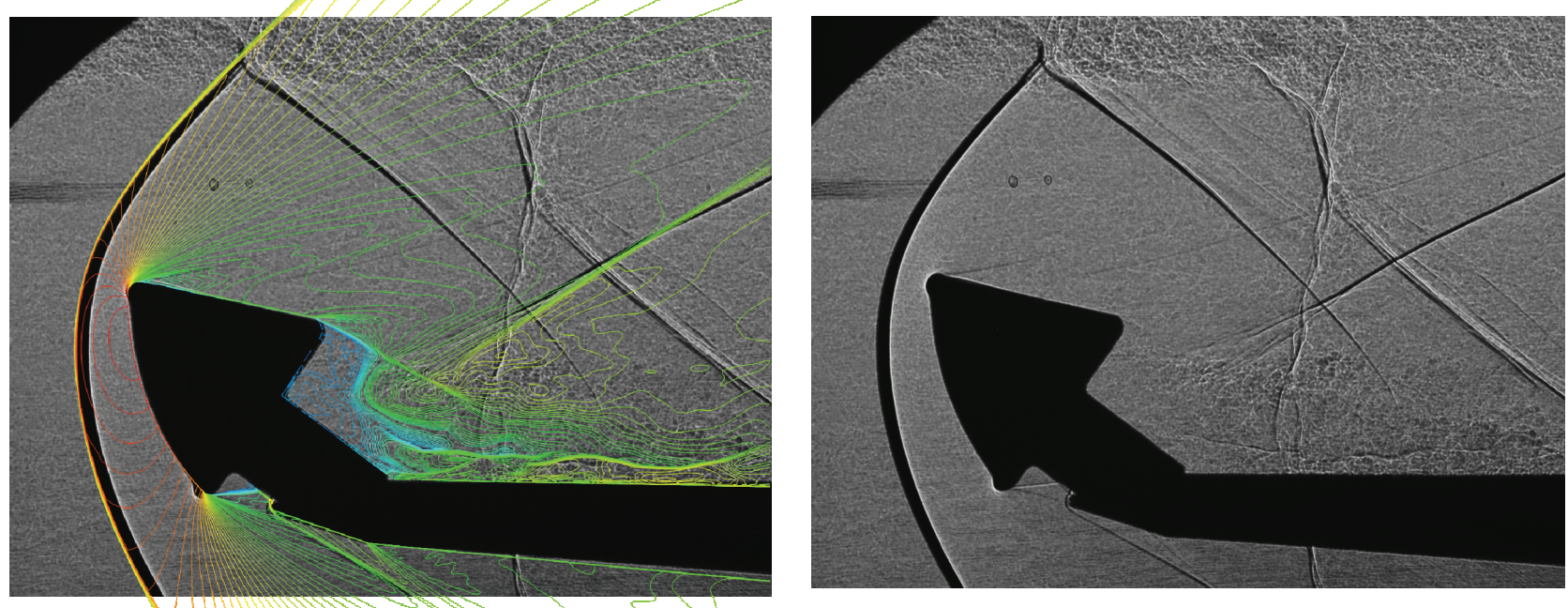

Figure 14: Mach 2, Angle of attack $22 \mathrm{deg}, \mathrm{Pt}=27 \mathrm{Bar}, \mathrm{T} 0=285 \mathrm{~K}$ 

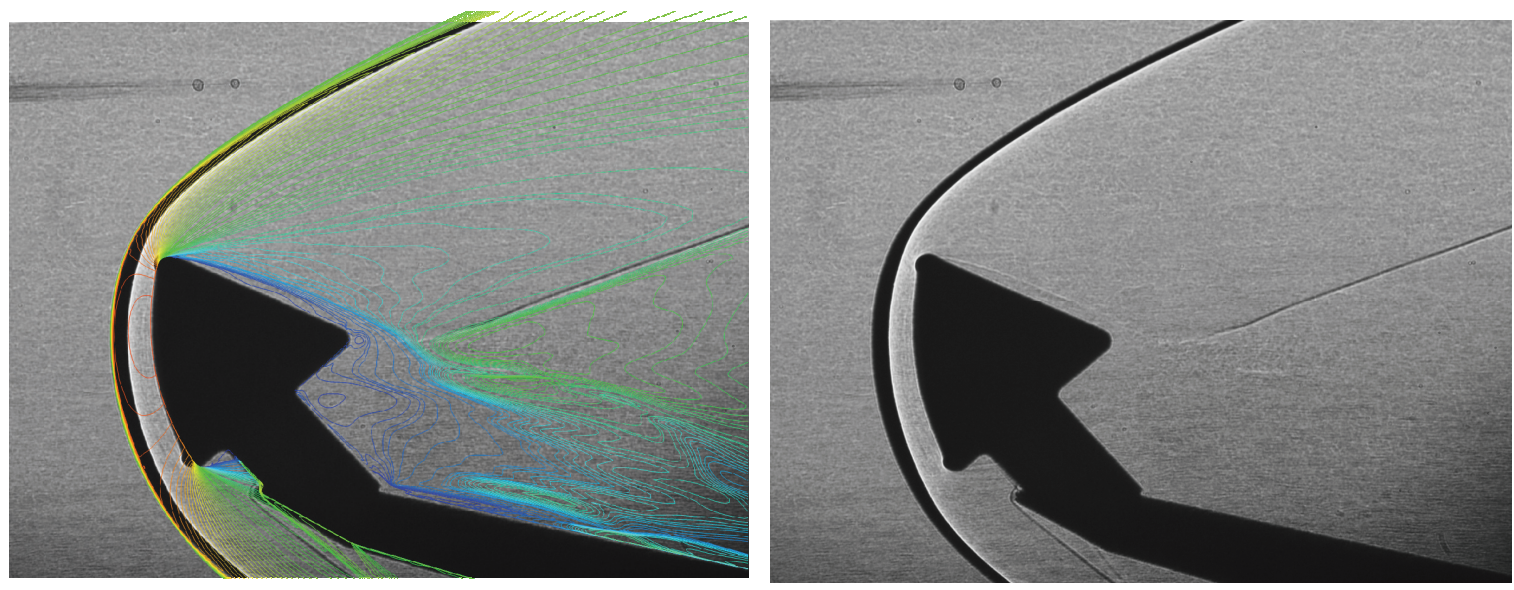

Figure 15: Mach 4, Angle of attack $10 \mathrm{deg}, \mathrm{Pt}=12 \mathrm{bar}$, T0=285K
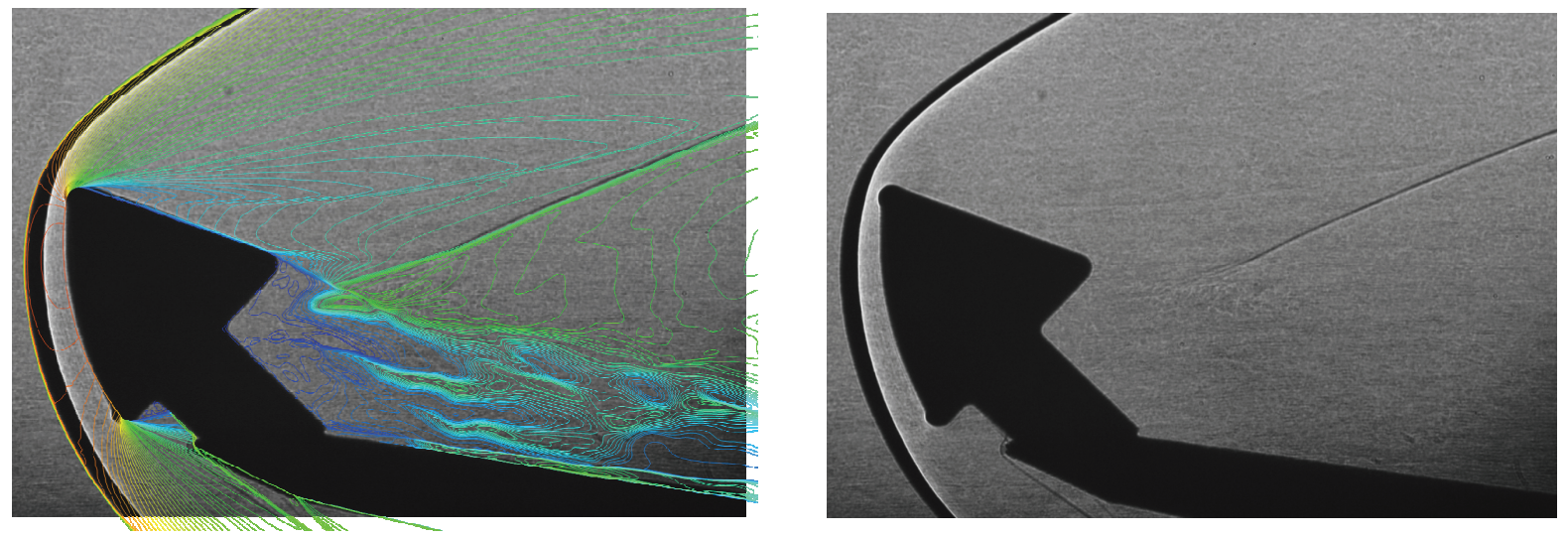

Figure 16: Mach 4, Angle of attack $14 \mathrm{deg}, \mathrm{Pt}=12 \mathrm{bar}, \mathrm{T0}=285 \mathrm{~K}$ 

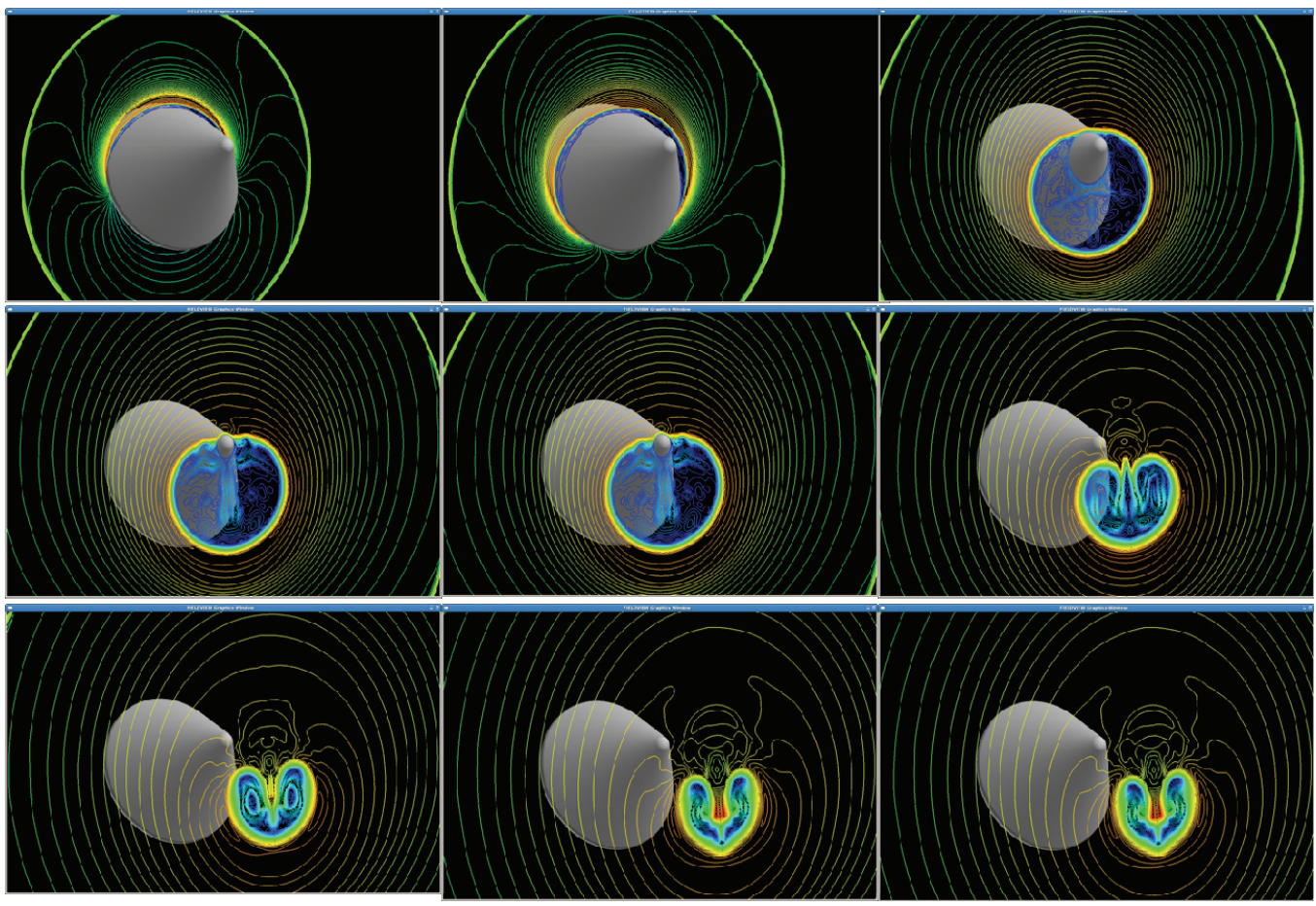

Figure 17 AS202 in TST27 (without blade) Mach 2 AoA 19 Mach contour x-cut planes computed with LORE, 8Mcells mesh

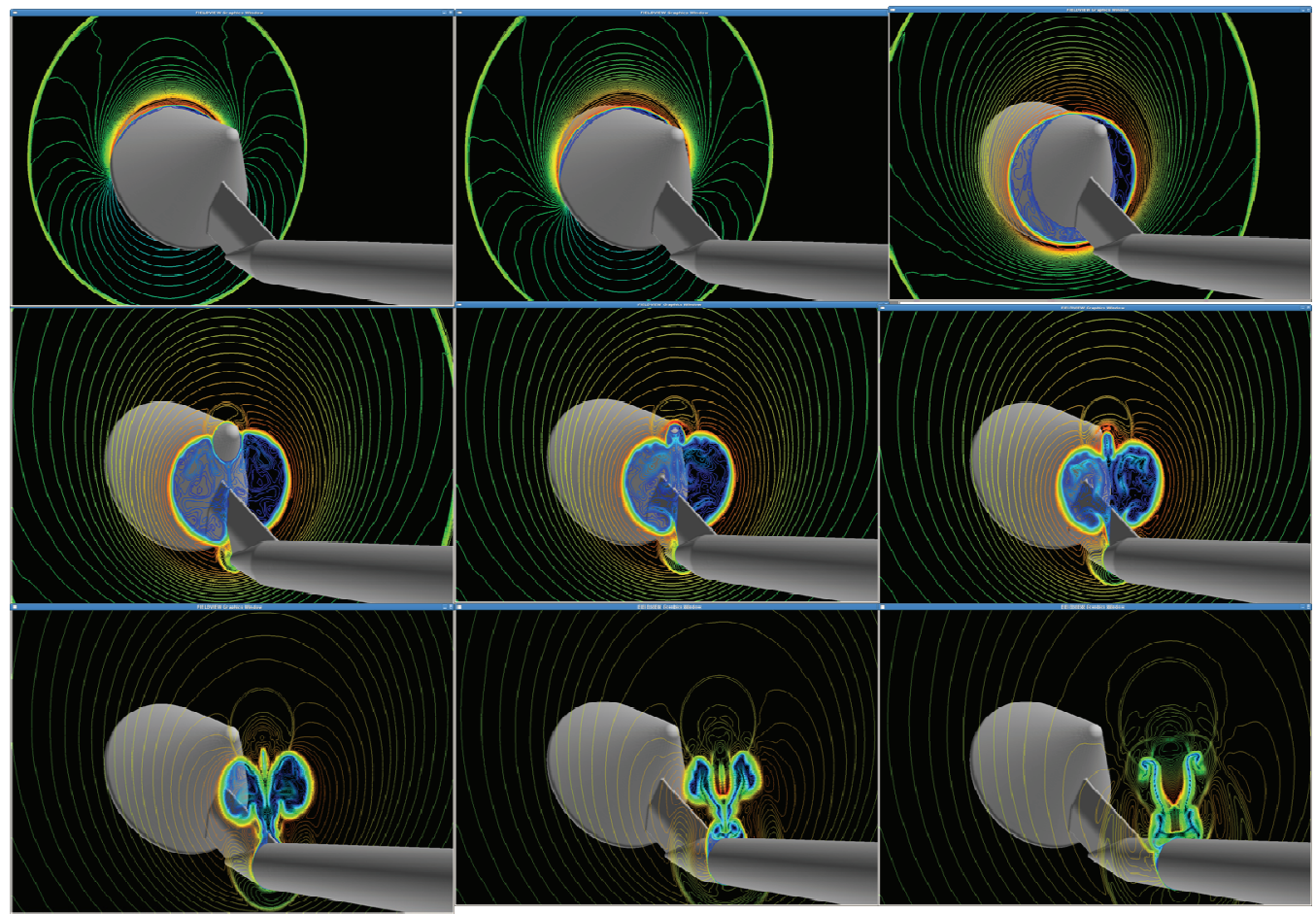

Figure 18 AS202 in TST27 (with blade) Mach 2 AoA 19 Mach contour x-cut planes computed with LORE: effect of sting / blade on 8Mcells mesh 


\section{B. AS-202 Flight Test Results and Related CFD Calculations}

In this section the comparison between CFD results obtained with different codes to measured afterbody heat fluxes during the AS-202 full-scale flight test is performed. A general discussion of the flow phenomena in the afterbody region is useful in order to understand the different flow conditions seen at the various calorimeter positions:

Due to the crossflow induced by the angle of attack two lobes corresponding to the off-axis trailing vortices are formed, and these twin-lobes extend much further aft in regions away from the pitch plane as these vortices separate in the wake. There are two large subsonic counter-rotating vortices on the leeside, and two more closely spaced smaller vortices below these past the rear apex. This is well reflected in the calculated skin friction stream lines at the AS-202 rear side shown in Figure 19 and also clearly evident for the calculated Mach 2 wind tunnel case (Figure 17). The large kidney-shaped areas of separated flow associated with this vortex pattern at the upper side of the base are clearly recognizable from the skin friction patterns. A smaller separation region extends from the upper side of the apex. This general separation pattern is well predicted by DPLR, LORE and TAU, with slight variations on the exact position of the separation lines, the LORE result being somewhat closer to the DPLR results than TAU (see Figure 20, 21).

The comparison between the computed afterbody heat transfer (assuming radiative equilibrium wall conditions with an emissivity of 0.85) in these flow areas and the experimental data for each of the 19 functional calorimeters "a - $\mathrm{s}$ " on the AS-202 Command Module is shown in Figure 22 (a) - (s). The agreement between the computations and the data is generally within the assumed experimental uncertainty for 15 of the 19 calorimeters. The calorimeters will be discussed in four separate groups: those on the shoulder in attached flow (" $h$ " and " $i$ ") those on the windward (attached) side of the afterbody (" $a-d "$, " $f$ ", and " $g$ "), those in the separated flow region ("e", " $m$ ", and " $o-s ")$ and those near the separation line (" $j ", " k ", " l "$, and " $n ")$.

On each plot in Figure 22, the flight data, which were scanned from Ref. 1, are shown as crosses. At some locations, particularly during the second heat pulse, there were multiple signal spikes. For example, notice the sharp heating spikes in the flight data on Figure 22(l) at around $t=4460$ and $t=4900 \mathrm{~s}$. These spikes were determined by Lee ${ }^{1}$ to correspond to the times of RCS firings, and thus are considered to be spurious. The data points that were estimated by the present authors to be spurious are shown on the plots as open circles. The best fit to the data for each case was then obtained using a Fourier-function based least-squares fitting procedure neglecting the spurious data points. Dashed lines indicate the assumed $\pm 20 \%$ uncertainty in the data ${ }^{4}$. For the purposes of this work the uncertainty was assumed to remain constant throughout the entry. Examination of Figure 22 indicates that the assumed uncertainty encompasses the majority of the data scatter, with the exception of some of the calorimeters in the separated flow region, which have more scatter during the skip maneuver ( $t=4600$ to $4800 \mathrm{~s}$ ). Finally, the CFD results at the trajectory points are shown as red diamonds for the DPLR analyses, as blue triangles for the LORE calculations (blue open triangles are used for a single calculation using a diffusion limited approach for modeling the wall catalycity) and as green diamonds for the TAU code results (unstructured grid results are shown as green open circle).

These results are now evaluated separately for the calorimeter positions in the shoulder region, the attached flow region at the afterbody, the separated flow region and separation line region on the afterbody. As reference, the findings concerning the DPLR results are taken from Ref. 4.

\section{Shoulder region}

Two calorimeters were placed on the shoulder just before the maximum diameter point. Calorimeter " $h$ " was placed midway between the windward and leeward centerline $\left(\theta=178.5^{\circ}\right)$, and "i" was placed on the leeward centerline $\left(\theta=270^{\circ}\right)$. Figure $22(\mathrm{~h})$ shows the comparison between the computed heating levels and the flight data for calorimeter "h".

DPLR results: Good agreement between the CFD and flight data. Peak heat flux predicted by the CFD is about $10 \mathrm{~W} / \mathrm{cm}^{2}$ at $\mathrm{t}=4510 \mathrm{~s}$, or about $6 \%$ higher than the flight data $(9.4 \mathrm{~W} / \mathrm{cm} 2)$. Agreement is generally within $20 \%$ over the entire trajectory, with the largest discrepancy occurring near the minimum between the two heat pulses 
( $\mathrm{t} \sim 4700 \mathrm{~s}$ ). Data for calorimeter "i" on the lee centerline is shown in Figure 22 (i): Once again the agreement is within the assumed $\pm 20 \%$ uncertainty throughout most of the flight.

LORE results: The LORE results are slightly below the DPLR results but also within the uncertainty range of the flight data for position "h". For position "i", the LORE results still are mostly within the uncertainty range falling below just after 4800s . Considering the large gradients in the shoulder region the CFD results compare surprisingly well to the measurements. The result of the single additional calculation using a diffusion limited approach to model wall catalycity (4800s) is nearly identical to the related result assuming super-catalytic walls. This is true for practically all sensor positions considered.

TAU Code results: TAU Code calculations were performed for two trajectory points $\mathrm{t}=4800 \mathrm{~s}$ and $\mathrm{t}=4900 \mathrm{~s}$. While the structured Level 1 (1Mio cells) meshes identical to the LORE meshes were used for both points in time, for the 4800 s case a hybrid/unstructured mesh was also employed to allow for comparisons of TAU solutions from structured and hybrid meshes.

The structured TAU results are practically identical to the LORE results for $t=4800 \mathrm{~s}$, however, for $\mathrm{t}=4900 \mathrm{~s}$ the TAU solution only matches calorimeter " $i$ ", and is at the lower end of the uncertainty band for calorimeter "h". The unstructured TAU solution at 4800 s is about $10 \%$ below the structured results for both sensor positions.

\section{Attached flow region}

Six calorimeters were placed on the conical afterbody on the windward side in a region where the flow remained attached throughout the entry. Calorimeters "a" through "d" were placed on or near the windward centerline, as shown in Figure 4. Calorimeter "g" was placed approximately midway between the shoulder and rear apex, at $\theta=$ $143^{\circ}$. The comparisons between the computed and experimental heat flux for these calorimeters are shown in Figure 22 (a) - (d) and (g).

DPLR results: From the figures we see generally good agreement during the first heat pulse. Heating levels near the peak heating point $(\mathrm{t}=4510 \mathrm{~s})$ are predicted to within $10 \%$ at all locations. Computed heating levels during the early portion of the second heat pulse also agree well with flight data, although the CFD results for calorimeters "c" and "d" at the final two trajectory points $(\mathrm{t}=4850$ and $\mathrm{t}=4900 \mathrm{~s})$ are lower than the flight data. The difference between the computation and flight data appears to increase with distance from the shoulder (the CFD results for calorimeter "c" are about $23 \%$ below the flight data at $\mathrm{t}=4900 \mathrm{~s}$, while those for calorimeter "d" underpredict flight data by $30 \%$ ). This may indicate that the flowfield is transitioning to turbulence on the attached afterbody during the second heat pulse. --- The computed heating at these calorimeters overpredicts the flight heating near the trough between the heat pulses, with the amount of overprediction near $\mathrm{t}=4700 \mathrm{~s}$ ranging from over $100 \%$ at calorimeter "a" to about $26 \%$ at calorimeter "g". There are several possible reasons for the CFD to predict higher heating than was measured in flight during this time period. During the period between about 4600 and 4800 seconds the spacecraft was undergoing a skip maneuver that resulted in a local maximum altitude at about 4700 seconds. During this skip phase local areas of non-continuum flow may have been present on the afterbody, which could result in an overprediction in heating. Also, during the high altitude skip phase of the entry, the uncertainty in vehicle orientation was much larger than average. In fact, between 4650 and 4750 seconds the uncertainty in angle of attack was approximately $\pm 2 \mathrm{deg}$., as opposed to $\pm 0.5 \mathrm{deg}$. during the remainder of the entry. It is possible that the low dynamic pressure during this portion of the trajectory could have prevented the vehicle from maintaining its trim orientation, resulting in a slightly smaller than expected angle of attack. If the angle of attack were small enough the flow could separate on the lee side of the afterbody, significantly reducing the predicted heating.

Calorimeter "f" (Figure 22 (f)) was placed near the rear of the shoulder at $\theta=138^{\circ}$. For this calorimeter, the CFD predictions are uniformly $33-50 \%$ higher than the data. Given the level of agreement seen for the other five calorimeters in this region, as well as that seen for the two calorimeters on the shoulder, the reasons for this disagreement are not clear.

LORE results: Apart from $\mathrm{t}=4900 \mathrm{~s}$, the LORE results are well within the uncertainty range for all sensor positions with the exception of the trough area discussed above (albeit the LORE results are somewhat nearer to the flight data in this area than the DPLR results). For $4900 \mathrm{~s}$, turbulent calculations using the Baldwin-Lomax as well as the Menter's SST model were also performed. These results come out within the upper region of the uncertainty area for calorimeter "d", but somewhat above upper fringe of the uncertainty area for calorimeter "c". This points to a possible turbulent flow situation in this area, not fully developed (transitional).

16

American Institute of Aeronautics and Astronautics 
TAU Code results: The laminar TAU-Code results are again close to LORE's. This is true for the structured grid as well as the unstructured grid results. In general, the TAU solutions on structured and unstructured meshes tended to be nearly identical in the attached flow regions, whereas deviations between results from structured and unstructured grids were found primarily in the separated flow regions.

For 4900s, an additional turbulent calculation using the Spalart-Allmaras model delivered heat fluxes well within and at the lower fringe of the uncertainty range for calorimeters "c" and "d".

In summary, all turbulent CFD calculations presented support the assumption of developing/transitional turbulent flow conditions in the area of the sensors in question. In view of the freestream Reynolds number all flight conditions considered are assumed to be laminar. Although for point (c) and (d) on the windward side, the flight data are hinting towards a turbulent condition, taking into account that the energy in the flow is less in the second peak as shown with the Fay-Riddell results, which is elaborated later in the paper. Possible reasons for transition could be protuberances, deteriorated walls or the promotion of transition due to crossflow ${ }^{10}$. An assessment was made to verify the laminarity of the base low with a transition criterion, commonly used in projects, in which the Reynolds number based on the momentum thickness over the Mach edge number, $\operatorname{Re}_{\Theta} / \mathrm{M}_{\mathrm{e}}$ is below 120. In Figure 25 the $\mathrm{Re}_{\Theta} / \mathrm{M}_{\mathrm{e}}$ is plotted for DPLR and LORE over the base region. A large part of the windward base is well below 120. Note that the computed values in a separated flow should not be taken into account since it is impossible to define the boundary layer edge there.

Turbulent augmentation factors resulting from the laminar solutions for selected sensor positions are contained in Table 4 for all turbulent solutions at $\mathrm{t}=4900 \mathrm{~s}$ : Turbulent heat flux results using DPLR (Baldwin-Lomax model) were reported in Ref. 4 for two sensor positions "c" and "d". Turbulent heat fluxes calculated with LORE (BaldwinLomax model and SST model) and with TAU (Spalart-Allmaras model) are given for seven sensor positions "a - d" and "h". The Baldwin-Lomax model predicts the highest turbulence levels, closely followed by the SST model. As seen for sensor position "c" and "d", the augmentation factors of the Baldwin-Lomax model compare well between DPLR and LORE. The lower augmentation factors of the Spalart-Allmaras model indicate a not fully developed turbulent flow. The Spalart-Allmaras turbulence model is known to predict fully turbulent flow only gradually after a transitional length. Therefore, the lower turbulent augmentation factors hints at a transitional flow prediction. It can be seen that both the full turbulent values resulting form the Baldwin-Lomax, SST and the transitional Spalart Allmaras model are within the uncertainty of the flight data. In summary, the flight uncertainty encompasses all turbulent CFD heating levels, whether transitional or fully turbulent, in the area of the concerned sensors.

\section{Separated flow region}

Seven calorimeters were placed in a region for which the flow remained separated during the entire heating portion of the entry.

DPLR results: Computational results for five of these, calorimeters " $m$ " and "o-r" are in generally good agreement with the flight data (see Figure 22. (m) and (o)- (r)). At each of these locations the peak heat flux during each pulse was between $0.2-0.4 \mathrm{~W} / \mathrm{cm}^{2}$. Agreement between the simulation and flight data was generally within $15 \%$ during the first heat pulse and the trough between the pulses. In contrast, the CFD prediction was generally lower than the flight data during the second heat pulse, which could be a consequence of turbulent transition. Note that the computed flow in the separation region became unsteady after $t=4850 \mathrm{~s}$. Therefore the computational results for 4850,4875 , and 4900 seconds include "error" bars, which attempt to bound the unsteadiness of the computed heat transfer.

The results for calorimeter "p" require further discussion. This calorimeter is located in close proximity to "o" (see Figure 22), and the computational results predict very similar heat fluxes for each. In contrast, the flight data indicate that the peak heating levels at calorimeter "p" were significantly higher than those at "o". As a result the CFD under predicts the heating at calorimeter " $\mathrm{p}$ " by about $45 \%$ at $\mathrm{t}=4530$ s, while the prediction at calorimeter "o" is well within the data scatter. This apparent discrepancy can be explained by examination of the after body layout in Ref. 1. While calorimeter "o" is mounted on a smooth area of the heat shield, calorimeter "p" was placed 
immediately in front of one of the rendezvous windows. Given this, it seems likely that the window created a local flow disturbance that affected the heat flux measured by calorimeter "p".

Calorimeter "e" was placed at the rear apex of the aeroshell. As seen in Figure 22 (e), the computations agree well with the flight data early in the first heat pulse and during the trough, but the computations significantly underpredict the peak heating levels. At $t=4530$ seconds the CFD result is about $45 \%$ lower than the flight data indicate. The disagreement during the second heat pulse can possibly be due to turbulent transition, but the differences in the first pulse are more difficult to understand. One possibility is that the local geometry of the apex is not accurately modeled in the current simulations.

By far the poorest agreement between the flight data and the CFD occurs at calorimeter "s" Figure 22 (s), which is near the rear apex on the leeward side near the centerline. At this location the flight data indicates heating levels nearly as high as those at the apex (calorimeter "e"), and slightly higher than those observed at calorimeter "d", which was at the same x-location but near the windward centerline. The computations predict very low heating levels at this location, consistent with those in the rest of the separated flow region. The reasons for this disagreement are not clear. Physically, the apex should create a separated flow region immediately behind it that would result in significantly lower heat transfer at calorimeter " $s$ " than either " $e$ " or " $d$ ". This relation is consistent with all CFD predictions although the absolute values are far off. This difference in absolute values can be only explained by a different local flow structure in the CFD solutions.

LORE results: The LORE calculation are generally significantly closer to the flight data for the second heat pulse than the DPLR results, albeit both LORE and DPLR also remain in some cases below the uncertainty band of the flight data.

TAU Code results: The TAU results capture the second heat pulse at 4900 for sensors "o" (within 10\% above the uncertainty limit ) and "p" (nominally). On the other hand the heatflux at $t=4800 \mathrm{~s}$ is overpredicted by TAU. At sensors "q" and "r" the TAU results match the flight data at $4800 \mathrm{~s}$ but are significantly lower at $4900 \mathrm{~s}$, near the lower limits of the "unsteadiness" error bars applied to the DPLR results. It must be underlined however, that in this low heatflux region, absolute heatflux density deviations in the order of just $1 \mathrm{~W} / \mathrm{cm}^{2}$ are of concern. Such low variations are considerably more challenging to model, considering the relative error bands (heat flux levels in the attached flow regions are one or two order above the heat fluxes typical for the separated regions). Interestingly, for the apex region "s", the best agreement between CFD and flight data is obtained wit the TAU code. This can be explained by the somewhat different separation line pattern predicted by TAU in that area..

When comparing the results of the three different codes it should be noted, that there are geometric differences between the apex as modeled and the flight vehicle that could result in different fluxes. There is also the possibility of a non-laminar heat flux condition in this region, which could lead to significantly higher fluxes. This could be attributed to a deteriorated wall or transition promotion due to crossflow.

\section{Separation line region}

Four calorimeters $(" \mathrm{j}, \mathrm{k}, \mathrm{l}, \mathrm{n} ")$ were placed in locations that were very near the separation line. Because the separation point is a function of Reynolds number, these calorimeters were in attached flow during a portion of the trajectory, and separated flow during the remainder.

DPLR results: The agreement between the computations and the flight data for these calorimeters was also generally good throughout the entry, as seen in Figure 22(j)-(l) and (n). The clearest evidence of transition from attached to separated flow can be seen at calorimeter " $j$ ", where the CFD shows sudden jumps in computed heating between $t=$ 4560 and $4600 \mathrm{~s}$ and again between $t=4750$ and $4800 \mathrm{~s}$ as the flow at this location attaches and then separates again. Similar jumps can be seen in the raw flight data at this location around $t=4600$ and $4750 \mathrm{~s}$ (see Figure 22(j)), although the levels are $20-30 \%$ lower than that predicted by the CFD. The computation also shows a jump in heating at calorimeter " $k$ " at $t=4700 \mathrm{~s}$ that corresponds to a transition from separated to attached flow. However in this case the scatter makes it impossible to determine whether a corresponding event was seen during the flight. Transition 
from a separated to an attached flow state is not readily visible in the surface heating at the other two calorimeter locations, however the general good agreement between the computations and the flight data indicates that the extent of separation is accurately predicted in the current simulations.

LORE results: For the sensors near the separation lines LORE/DPLR are alternatively closer to the flight data during certain trajectory phases: this is especially noticeable for the data points towards the end of the trajectory, after $\mathrm{t}=4800 \mathrm{~s}$, at sensor positions " $\mathrm{j}, \mathrm{l}$ " and " $\mathrm{n}$ ", were the LORE and DPLR results tend to diverge. This underlines the fact that for those calorimeter positions, minor differences in the determination of the separation line positions between the codes can have a significant impact.

TAU Code results: TAU results alternate between heat flux values nearer at DPLR or LORE data, depending on the time and sensor position. Again differences in the precise determination of the separations lines can be expected to show a significant impact on the individual results.

In summary, throughout most of the flight trajectory points considered, the afterbody heat fluxes are predicted within the uncertainty range of the flight measurements by all three CFD Codes DPLR, LORE and TAU, in spite of the fact that different wall catalycity models, reaction rate constants, and structured as well as unstructured CFD meshes were employed between the codes, which result in differences among the CFD results. As expected, larger deviations between the codes and flight data occur in separated flow areas.

\section{Evaluation of semi-analytical, approximate engineering approaches for the assessment of afterbody heat fluxes:}

As an alternative approach, the LORE and TAU CFD results obtained for 4800s and 4850s, resp, has been used to approximate the sensor heat fluxes vs. time over the complete trajectory range by scaling the CFD generated heat fluxes for the freestream velocity and density at $4800 \mathrm{~s}\left(\mathrm{~V}=6210 \mathrm{~m} / \mathrm{s}, \rho=0.000137 \mathrm{~kg} / \mathrm{m}^{3}\right)$ with the freestream densities and velocities associated with all other trajectory points of interest via the simple relationship (Fay-Riddell stagnation point analysis):

$$
Q(t) / Q(4800 s)=[\rho(t) / \rho(4800 s)]^{0.5} \times[V(t) / V(4800 s)]^{3}
$$

This simplified approach is often used in project work to approximately determine the heat flux evolution on reentry vehicles from just one or two (comparatively expensive) Navier-Stokes CFD solutions over the whole trajectory.

The heat fluxes for sensor positions "a, b, g" and "h" on the windward afterbody side in comparison to the flight data and to the full range of DPLR CFD results is shown in Figure 23 The curves in Figure 23 basically underline for the current case the validity of this engineering approach in a conservative sense, considering that the maximum absolute heat flux peaks tend to be higher than the flight measurements, albeit the maximum between left and right peak is in some cases interchanged compared to the flight measurements. However, it is evident that the local accuracy here depends heavily on the flow region, sensor " $\mathrm{h}$ " showing a significantly better correlation to the flight data (fully within the uncertainty range throughout the trajectory) than the other sensors shown. However, at all sensor positions shown, an area in the middle of the heat flux peaks falls within the uncertainty range of the flight data.

Since the data are basically scaled with respect to the stagnation point heat flux using this engineering approach, the general trend of the heat fluxes on all positions on the surface is implicitly assumed to follow the trend of the stagnation point heat flux which obviously does not work out equally well for all positions at the afterbody surface.

The relation of measured base flow heat fluxes (colored curves) vs. flight time at all calorimeter positions to the stagnation point heat fluxes can be found in Figure 24. The stagnation point heat flux density of AS202 was determined through the Fay-Riddell formula without correction for the angle of attack (black curve in Figure 24). 
Now Figure 24 shows that the measured heat fluxes at the majority of the 19 calorimeter positions remain at or below 7\% of the Fay-Riddell stagnation point heat flux throughout the trajectory. Only 3 calorimeters " $h$ ", "i"and "f", all positioned at or very close to the shoulder, recorded higher heat flux levels going up to $26 \%$ of the FayRiddell heat fluxes in the case of "h"

Even if the $20 \%$ uncertainty on top of the measured flight data is applied, it can be concluded that the assumption of the base heat fluxes nowhere exceeding $\sim 10 \%$ of the Fay-Riddell heat flux represents a conservative engineering estimate for the Apollo shape. Accordingly, just in the shoulder region of the afterbody, application of $\sim 35 \%$ of the Fay-Riddell heat fluxes seems to provide a conservative engineering estimate.
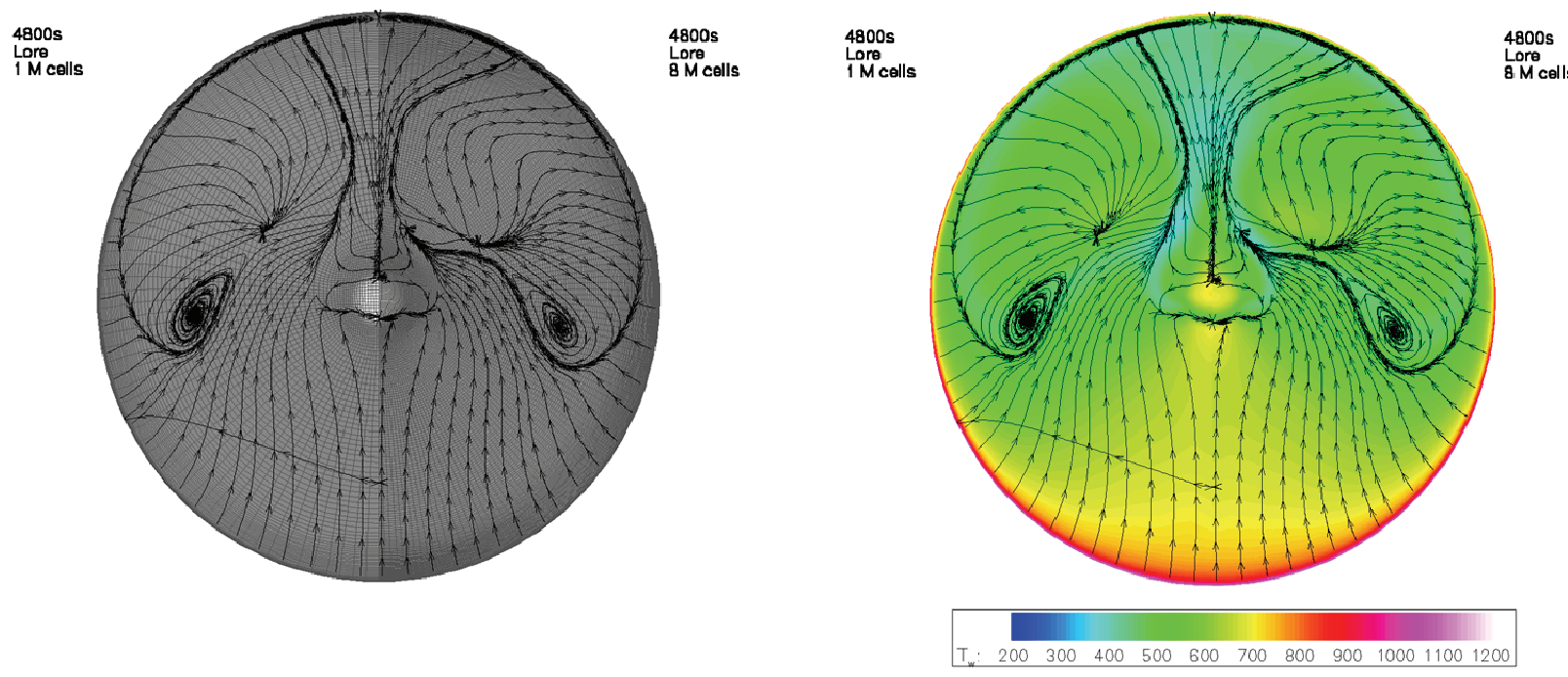

Figure 19. Surface temperatures and skin friction stream lines on the back of the AS-202 at 4800s: influence of grid refinement by doubling the mesh in 3 directions

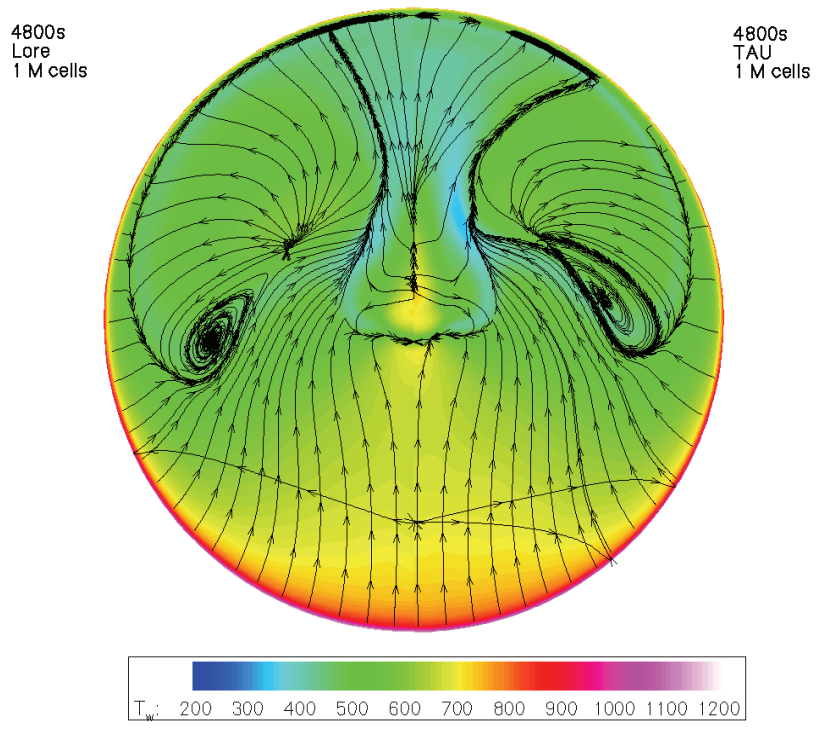

Figure 20 Surface temperatures and skin friction stream lines on the back of the AS-202 at 4800s: comparison LORE -TAU

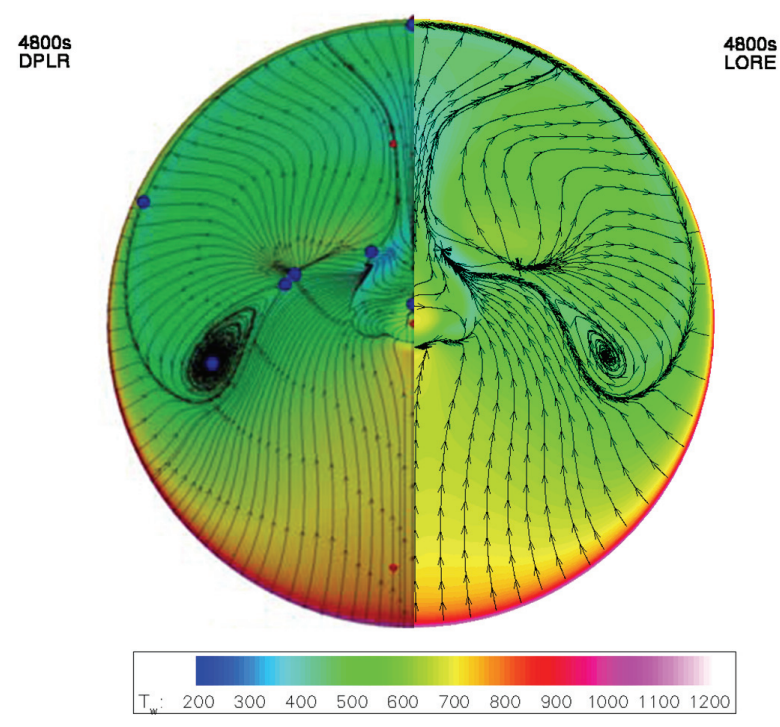

Figure 21 Surface temperatures and skin friction stream lines on the back of the AS-202 at 4800s: comparison LORE -DPLR 


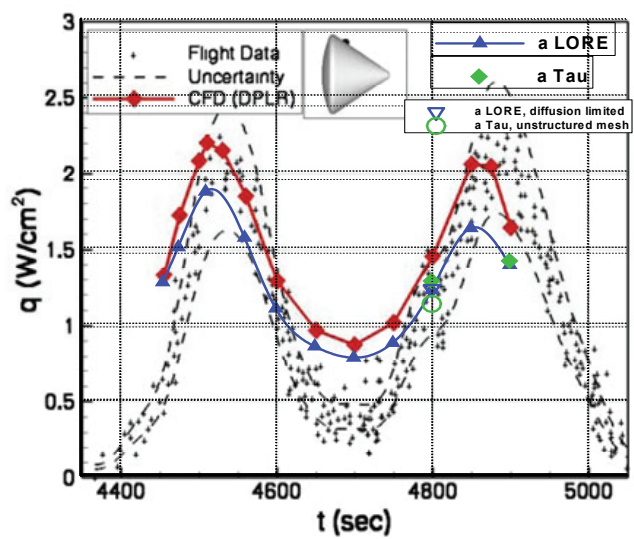

Calorimeter (a)

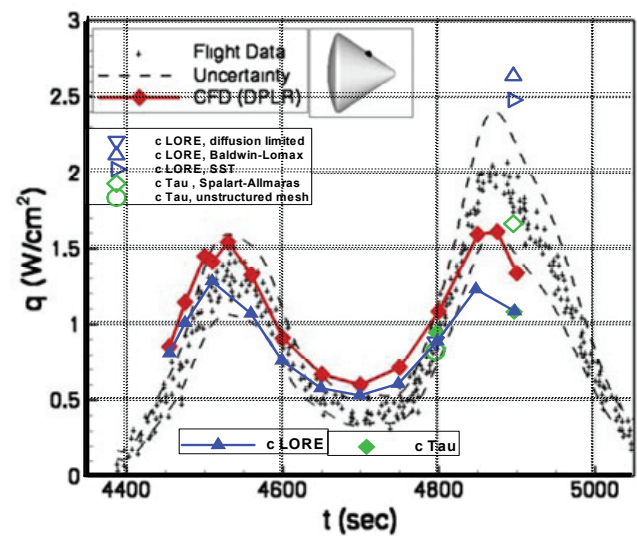

Calorimeter (c)

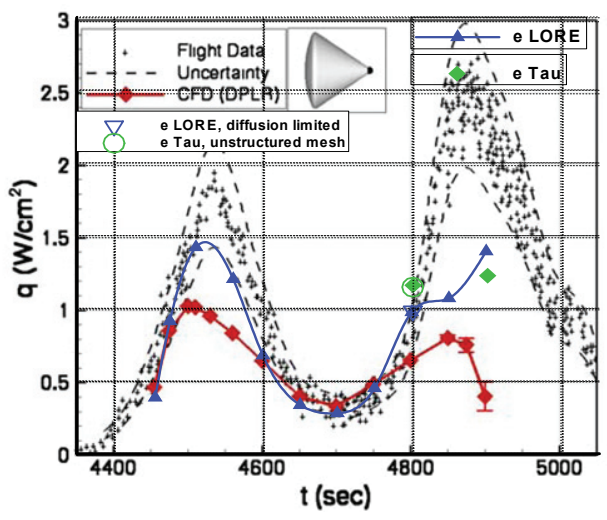

Calorimeter (e)

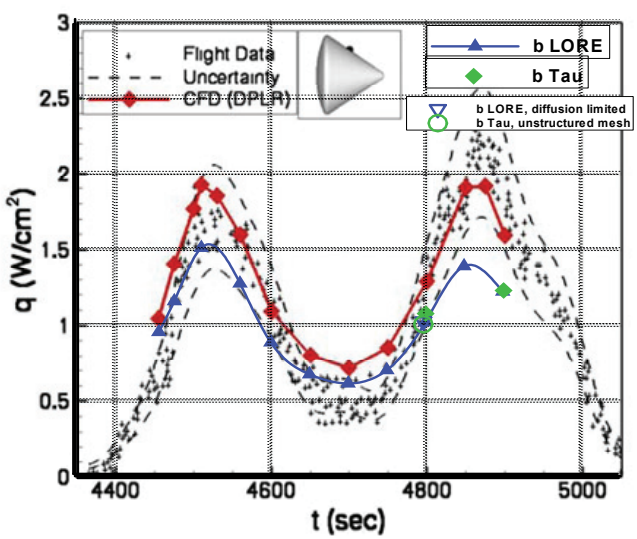

Calorimeter (b)

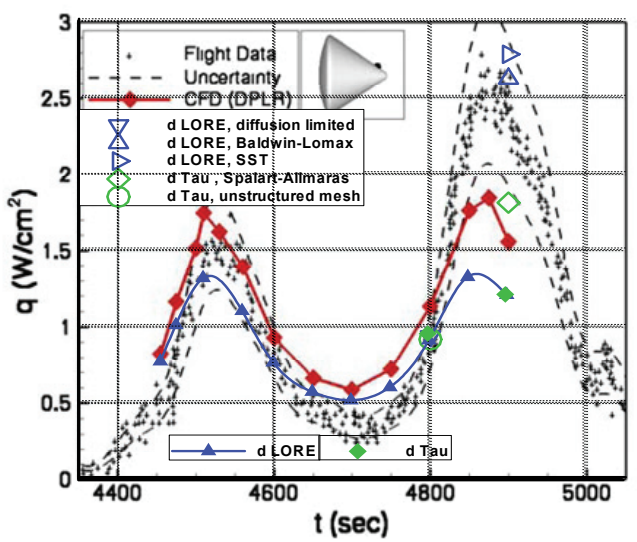

Calorimeter (d)

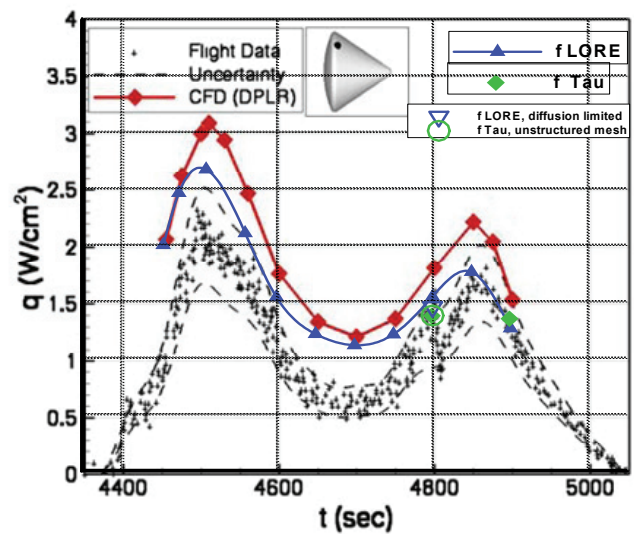

Calorimeter (f)

Figure 22. Comparison of computed and experimental heat transfer for AS-202. 

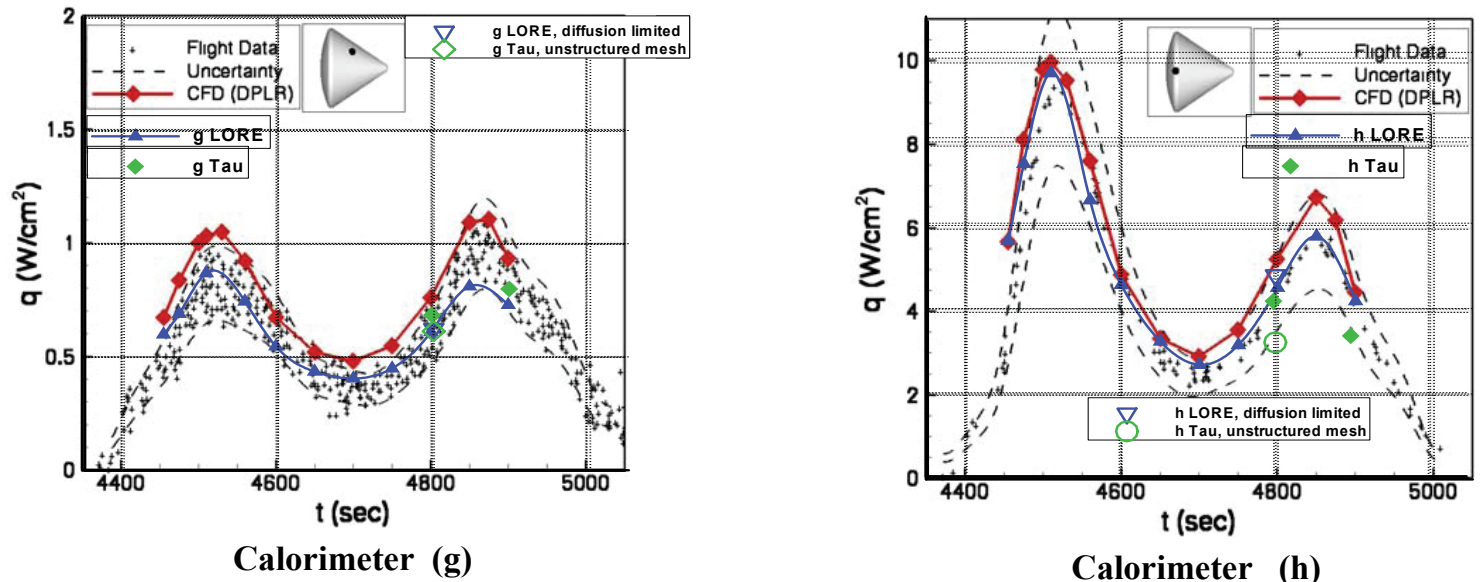

Calorimeter (h)

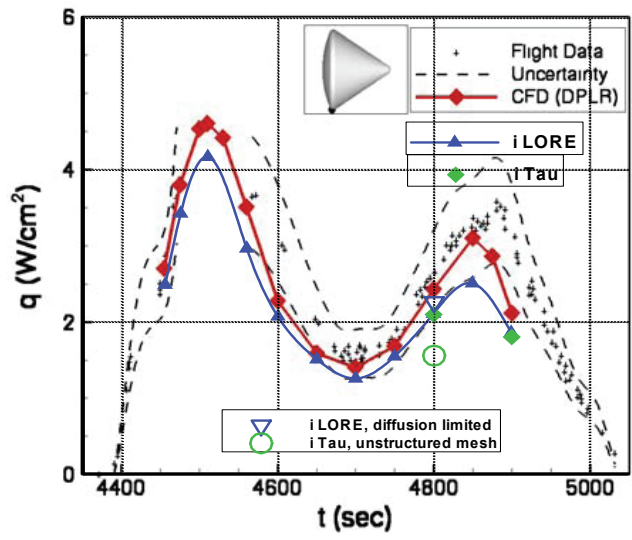

Calorimeter (i)

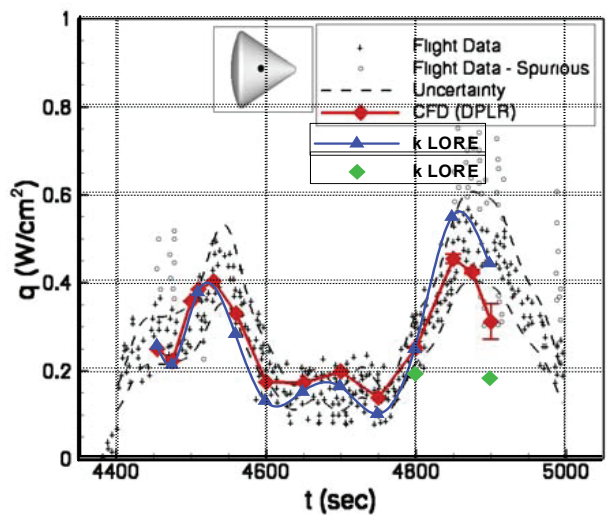

Calorimeter (k)

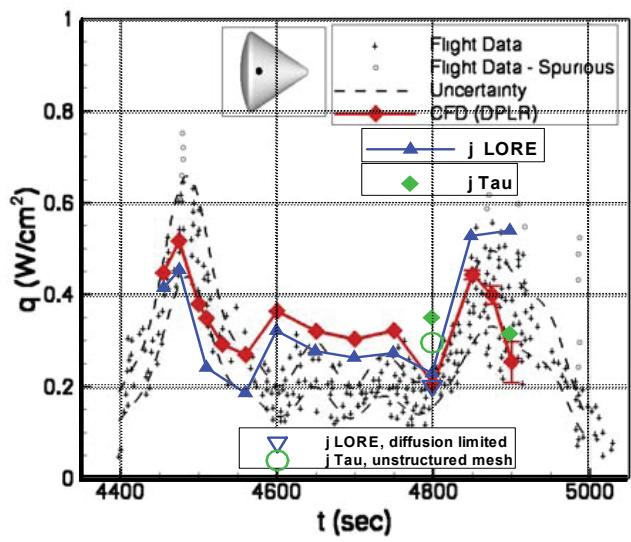

Calorimeter (j)

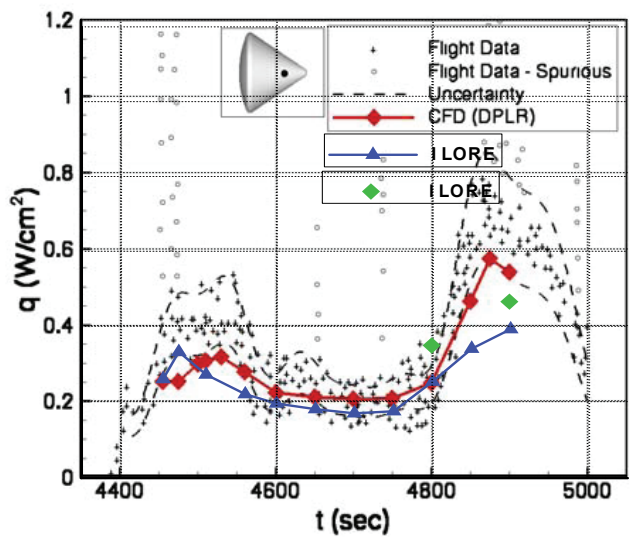

Calorimeter (I)

Figure 22. Comparison of computed and experimental heat transfer for AS-202, continued. 

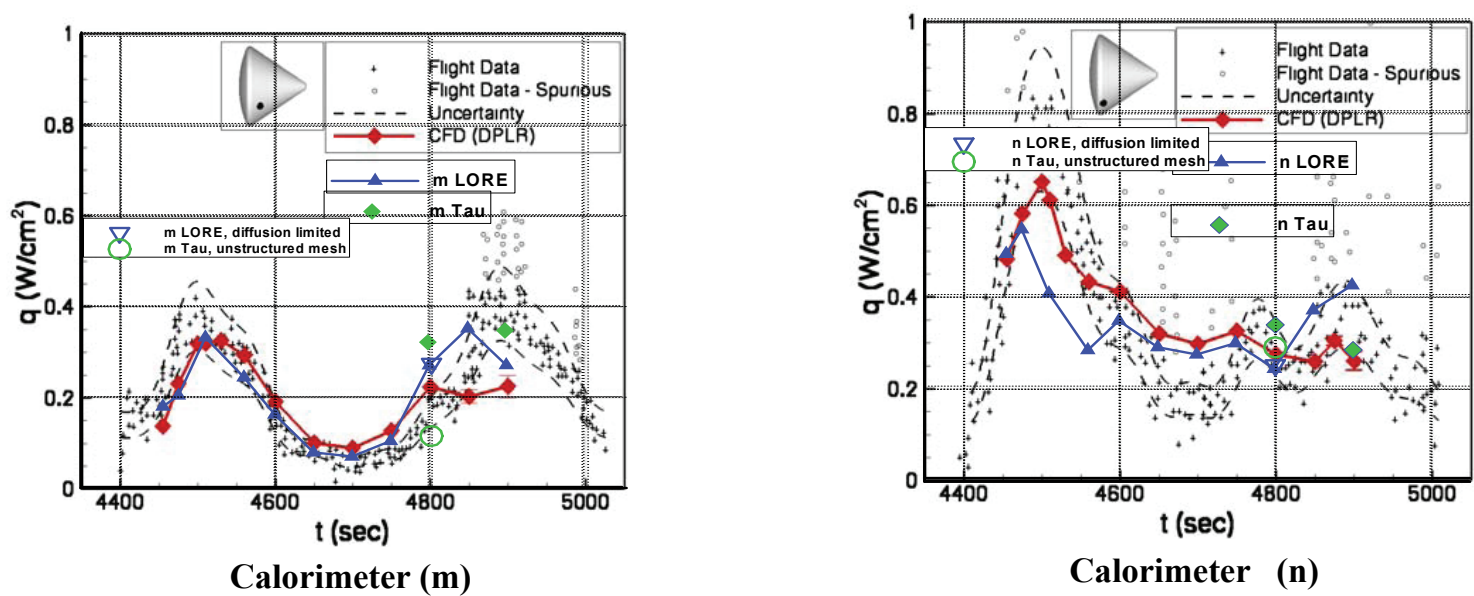

Calorimeter (n)
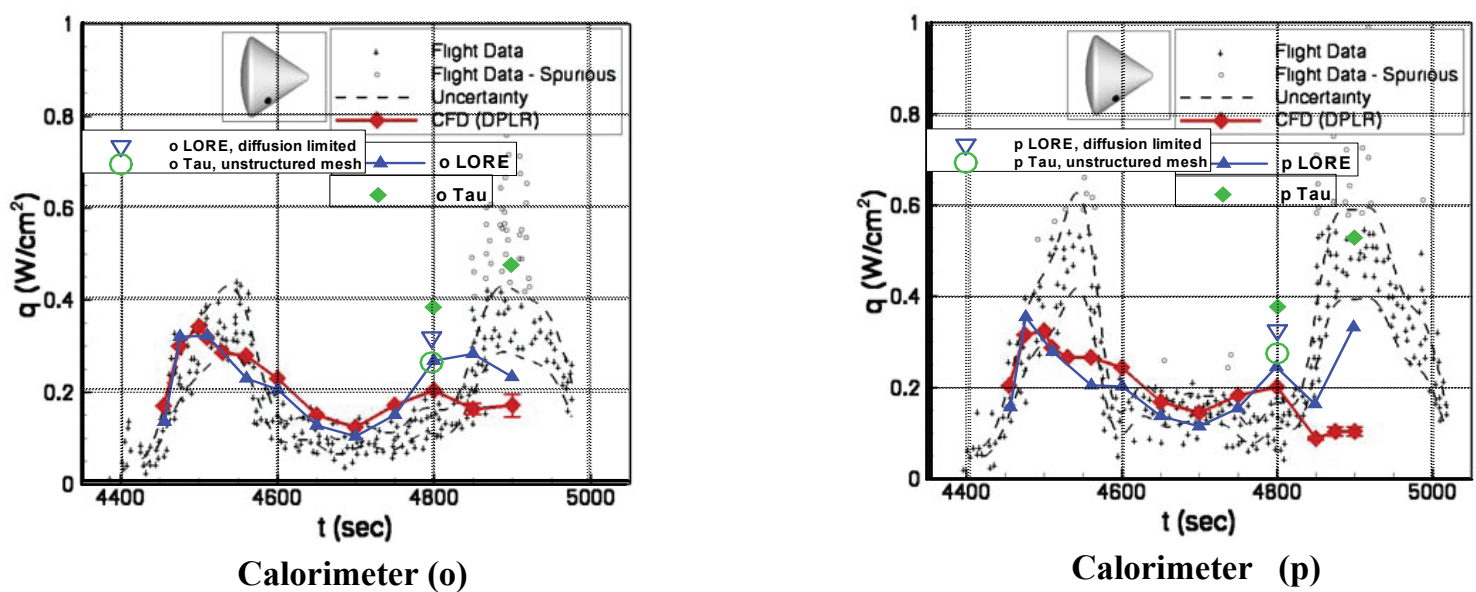

Calorimeter (p)

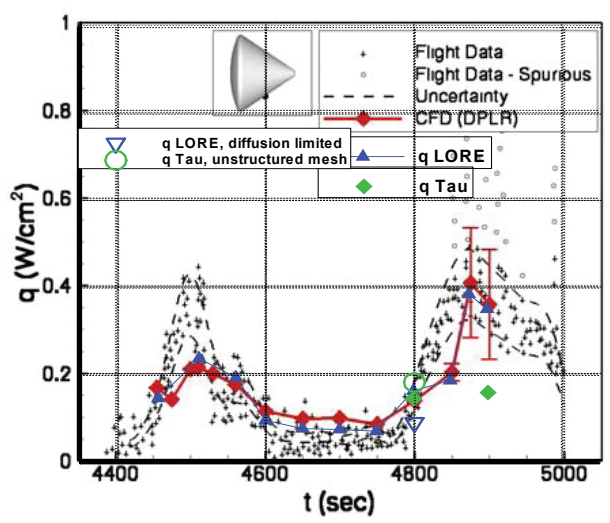

Calorimeter (q)

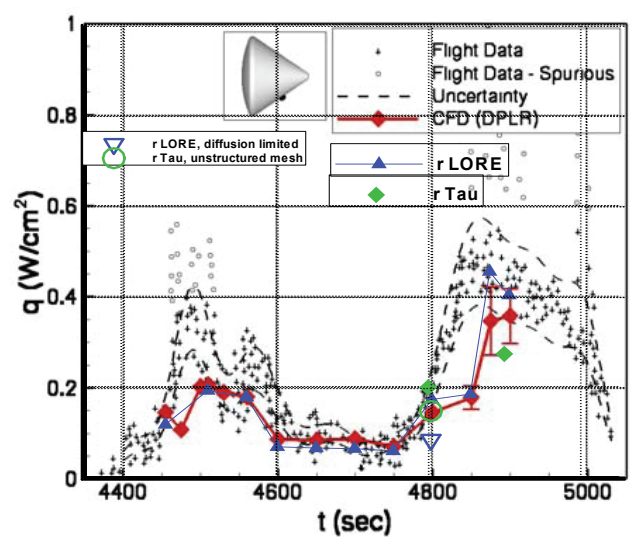

Calorimeter (r)

Figure 22 Comparison of computed and experimental heat transfer for AS-202, continued. 


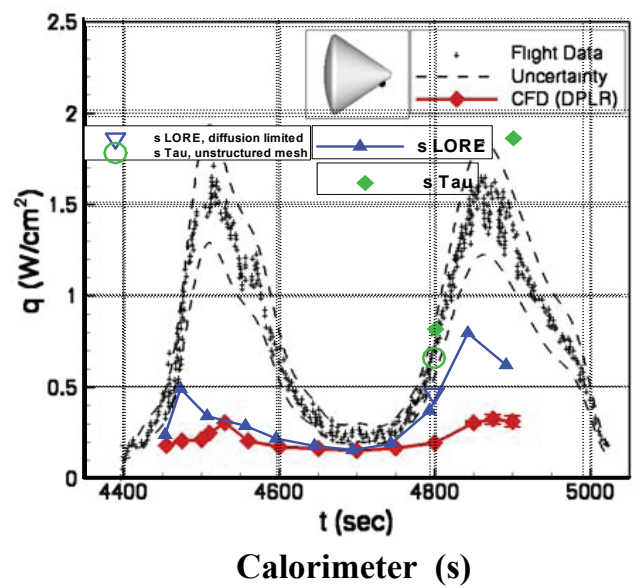

Figure 22. Comparison of computed and experimental heat transfer for AS-202, continued
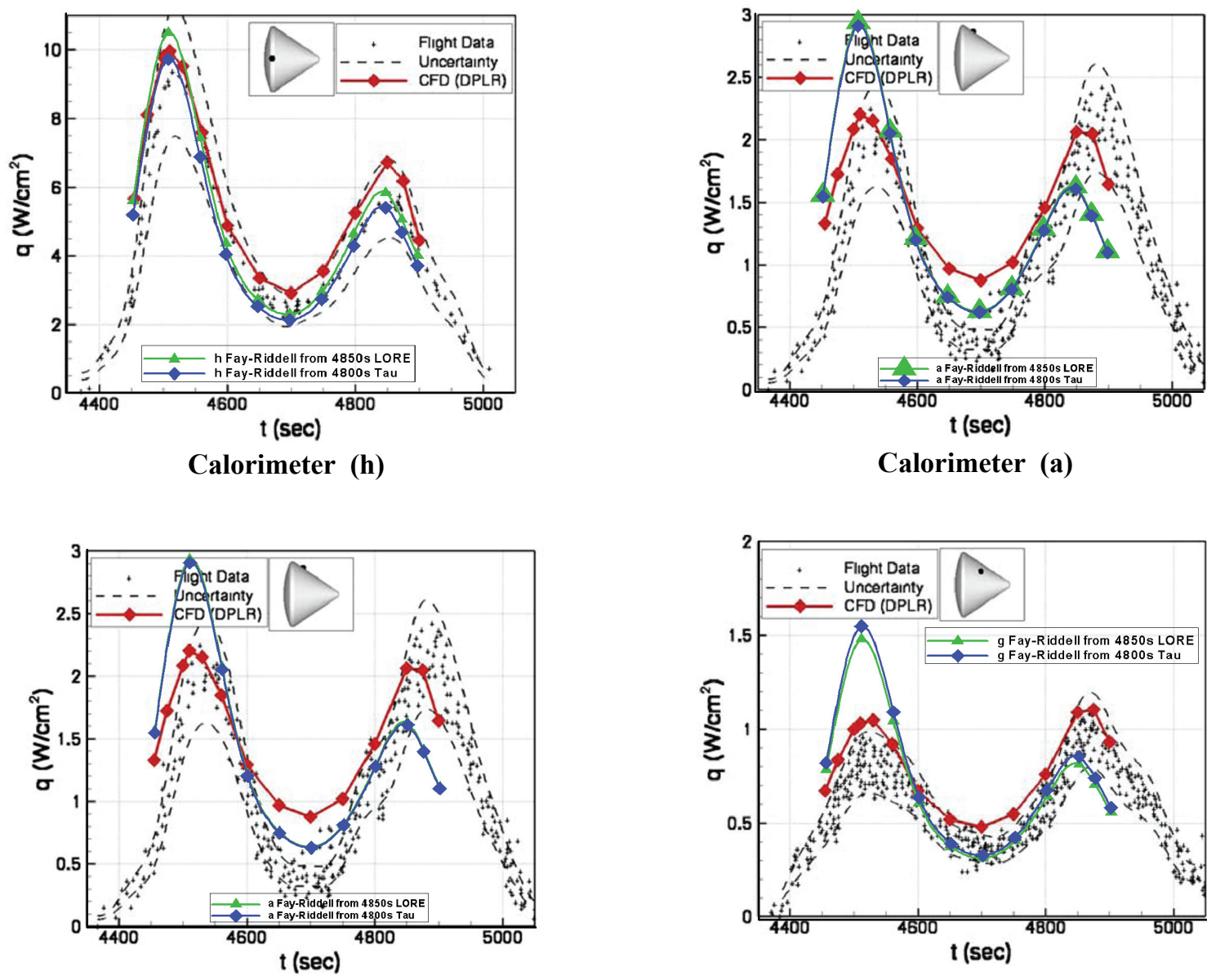

Calorimeter (b)

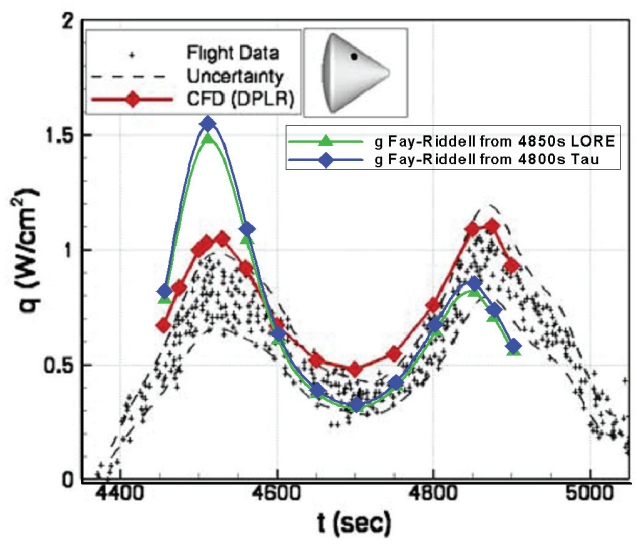

Calorimeter (g)

Figure 23. Heat flux time history on windward side of the AS202 base computed with Fay-Riddell anchored with one CFD analysis 


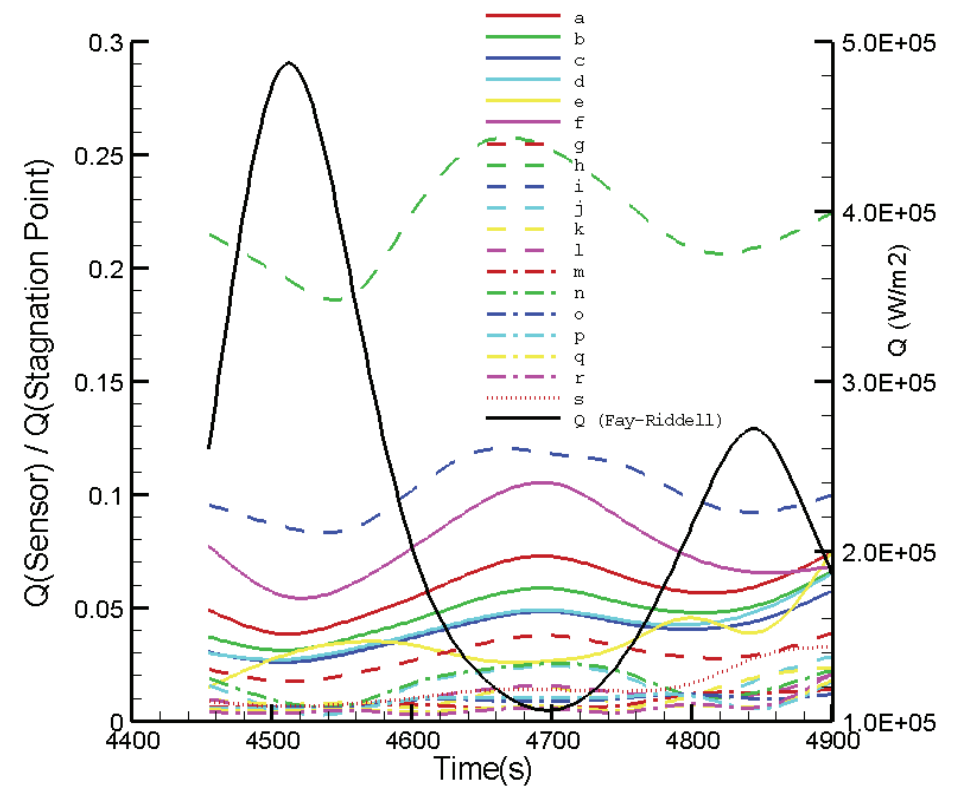

Figure 24: Analytical heat flux prediction / Relation of measured base flow heat fluxes to Fay-Riddell stagnation point heat flux .

DPLR

LORE

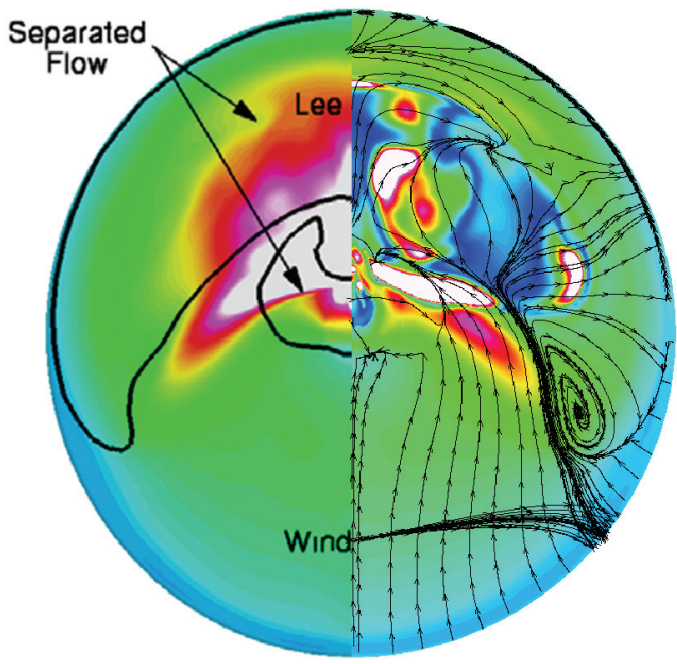

$\mathrm{Re}_{\mathrm{d}} \mathrm{M}_{\mathrm{e}}$

Figure 25: Comparison of computed transition parameter on the conical afterbody for $t=4900 \mathrm{~s}$. 
Table 4: AS-202 turbulent augmentation factor turbulence modes/codes for 4900s.

\begin{tabular}{ccccccc}
\hline \hline $\begin{array}{c}\text { calorimeter } \\
\text { Code/model }\end{array}$ & $\mathbf{a}$ & $\mathbf{b}$ & $\mathbf{c}$ & $\mathbf{d}$ & $\mathbf{g}$ & $\boldsymbol{h}$ \\
\hline TAU/lam & 1.22 & 1.07 & 1.21 & 1.24 & 3.36 & 1.79 \\
TAU/SA & 1.46 & 1.67 & 1.8 & 1.52 & 3.45 & 2.16 \\
Factor SA & $\mathbf{1 . 2 0}$ & $\mathbf{1 . 4 6}$ & $\mathbf{1 . 5 5}$ & $\mathbf{1 . 4 9}$ & $\mathbf{1 . 3 4}$ & $\mathbf{1 . 0 3}$ \\
& & & & & & \\
LORE lam & 1.23 & 1.07 & 1.21 & 1.39 & 4.18 & 1.86 \\
BL & 3.16 & 2.96 & 2.64 & 2.61 & 1.91 & 8.91 \\
Factor BL & $\mathbf{2 . 2 9}$ & $\mathbf{2 . 4 1}$ & $\mathbf{2 . 4 8}$ & $\mathbf{2 . 1 5}$ & $\mathbf{2 . 6 2}$ & $\mathbf{2 . 1 3}$ \\
& & & & & & \\
LORE SST & 2.31 & 2.68 & 2.49 & 2.82 & 1.27 & 5.55 \\
Factor SST & $\mathbf{1 . 6 7}$ & $\mathbf{2 . 1 8}$ & $\mathbf{2 . 3 3}$ & $\mathbf{2 . 3 3}$ & $\mathbf{1 . 6 7}$ & $\mathbf{2 . 1 8}$ \\
& & & & & & \\
DPLR lam & & & 1.8 & 2.4 & & \\
DPLR BL & & & $\mathbf{2 . 4 6}$ & $\mathbf{2}$ & & \\
Factor BL & & & & & & \\
\hline \hline
\end{tabular}

\section{Conclusion}

In order to assess the predictive capability of capsule base flows with hypersonic CFD codes, the AS-202 shape have been investigated in well controlled laminar supersonic wind tunnel flow conditions. Flight afterbody heating data of the Apollo AS-202, measured during a large portion of its re-entry has been used for the assessment of prediction capabilities in the presence of reacting flows.

To that end, a wind tunnel campaign has been performed in the TST27 facility at the TU Delft. The tests have provided reference data for the appraisal of current high fidelity hypersonic CFD codes used in most ESA entry projects. During the tests, flow patterns with particular emphasis on shear layer separation at the capsule shoulder were investigated for various combinations of angle of attack and Mach number. The CFD calculations performed showed an excellent agreement to the flow topologies observed during wind tunnel tests, in terms of capturing salient flow features: Bow shock, shoulder region, wake area Shock-shock interactions/flow interferences due to the presence of a wind tunnel blade mounting, local separation and reattachment of the capsule flow and characteristic flow patterns of the leeside/wake flow have been accurately reproduced.

The good predictive capabilities of the CFD codes, LORE, TAU and DPLR, involved in the comparison of AS202 in-flight afterbody heat flux measurements has been established, taking into account the large flight uncertainties and the very low levels of heat fluxes $\left(<10 \mathrm{~W} / \mathrm{cm}^{\wedge} 2\right)$. Relative deviations are still found in the calculated afterbody heat flux levels between flight data and CFD as well as between different CFD codes which tend to be largest in areas of separated flow, and sensitive to details of the different numerical schemes. However, in these areas the absolute heat flux levels tend to be comparatively small, i.e. even a small deviation in absolute terms has a greater impact on relative error.

Since the computed heat fluxes agreed well to the AS-202 flight data within the uncertainty for most of the calorimeters, the present design margin of $200 \%$ commonly applied to afterbody simulations, could be reduced. 


\section{Acknowledgments}

The authors would like to thank Rafael Molina of the European Space Agency for providing his support, Stan van Gemert, $\mathrm{COO}$, for enabling and managing the computational infrastructure at AOES and the CEO, Paul Pearson, for the support to the AVT making this work possible. Also many thanks to Greg Byshenk and Alessio Terpin, for building an optimized cost-performance cluster environment.

\section{References}

1 Lee, D.B., Bertin, J.J., and Goodrich, W.D., "Heat Transfer Rate and Pressure Measurements Obtained During Apollo Orbital Entries," NASA TN D-6028, Oct. 1970.

2 Lee, D.B, “Apollo Experience Report: Aerothermodynamics Evaluation,” NASA TN D-6843, Jun. 1972.

3 Hillje, E., "Entry Flight Aerodynamics from Apollo Mission AS-202," NASA TN D-4185, Oct. 1967.

4 Wright, M.J., Prabhu, D.K., and Martinez, E.R., "Analysis of Afterbody Heating Rates on the Apollo Command Modules, Part 1," Journal of Thermophysics and Heat Transfer, Vol. 20, No. 1, 2006, pp. 16-30.

5 Walpot, L., Development and Application of a Hypersonic Flow Solver, Ph.D. thesis, T.U. Delft University, May 2002.

6 Gerhold, T., O. Friedrich, J. E., and Galle, M., "Calculation of Complex Three-Dimensional Configurations Employing the DLR-TAU-Code," 16th Aerospace Sciences Meeting, Reno, NV, USA, January 1997, AIAA paper 97-0167.

7 L.M.G. Walpot, P. Noeding, F. Tarfeld, R.C. Molina, A.Gülhan, J.-C. Paulat, "Transonic and Supersonic Static Stability Analysis of the CARV Reentry Vehicle", 14th AIAA/AHI Space Planes and Hypersonic Systems and Technologies Conference, AIAA Paper 2006-8077, 2006

8 R.N. Gupta, J.M. Yos, R.A. Thompson, K.-P. Lee, "A Review of Reaction Rates and Thermodynamic and Transport Properties for an 11-Species Air Model for Chemical and Thermal Nonequilibrium Calculations to 30000K", NASA-RP-1232, 1990

9 Kruse, R., "Transition and Flow Reattachment behind an Apollo-like Body at Mach Numbers to 9", Technical Note D-4645, NASA, 1968.

10 Anderson John D., Hypersonic and high temperature gas dynamics, 1988 AIAA

11 Schrijer, F.F.J., and Walpot, L.M.G.F.M., "Experimental Investigation of the Supersonic Wake of a Reentry Capsule," 48th AIAA Aerospace Sciences Meeting, Orlando, Florida, January 2010, AIAA-2010-1251 